\title{
A numerical mapping of energy gains in a powered Swing-By maneuver
}

\author{
Alessandra F. S. Ferreira 1 \\ Antônio F. B. A. Prado • Othon C. Winter
}

Received: 25 October 2016 / Accepted: 15 March 2017 / Published online: 28 March 2017

(C) Springer Science+Business Media Dordrecht 2017

\begin{abstract}
The present paper studies the effects of a powered Swing-By maneuver, considering the particular and important situations where there are energy gains for the spacecraft. The objective is to map the energy variations obtained from this maneuver as a function of the three parameters that identify the pure gravity Swing-By with a fixed mass ratio (angle of approach, periapsis distance and velocity at periapsis) and the three parameters that define the impulsive maneuver (direction, magnitude and the point where the impulse is applied). The mathematical model used here is the version of the restricted three-body problem that includes the Lemaître regularization, to increase the accuracy of the numerical integrations. It is developed and implemented by an algorithm that obtains the energy variation of the spacecraft with respect to the largest primary of the system in a maneuver where the impulse is applied inside the sphere of influence of the secondary body, during the passage of the spacecraft. The point of application of the impulse is a free parameter, as well as the direction of the impulse. The results
\end{abstract}

A. F. S. Ferreira $(\varangle)$ · A. F. B. A. Prado Instituto Nacional de Pesquisas Espaciais, São José dos Campos, São Paulo 12227-010, Brazil

e-mail: aleferrazsilva@hotmail.com

A. F. B. A. Prado

e-mail: antonio.prado@inpe.br

O. C. Winter

Universidade Estadual Paulista, Guaratinguetá,

São Paulo 12516-410, Brazil

e-mail: ocwinter@gmail.com make a complete map of the possibilities, including the maximum gains of energy, but also showing alternatives that can be used considering particularities of the mission.

Keywords Astrodynamics - Swing-By - Close approach · Orbital maneuvers · Interplanetary missions

\section{Introduction}

The Swing-By maneuver is a strategy to give energy to a spacecraft by using a close approach with a celestial body. It modifies the velocity, energy and angular momentum of the spacecraft. There are several applications for this maneuver, with the most usual one the fuel economy in space missions. The powered SwingBy maneuver combines the pure gravity Swing-By with an impulse applied to the spacecraft near the close encounter. The literature [1,2] shows a comparison between two types of powered Swing-By: (i) a maneuver where the impulse is applied exactly during the passage of the spacecraft by the periapsis of its orbit around the celestial body; (ii) a maneuver where the impulse is applied after the spacecraft enters the sphere of influence [3] of the celestial body. This is done to verify the efficiency of both maneuvers, which means to find the best maneuver to be made.

Based in reference [1], Casalino et al. [4] showed a more flexible maneuver, where a constraint on the 
periapsis altitude replaces the fixed value used in reference [1], so leaving space to vary the point where the impulse is applied. The "patched-conics" model was used, and they showed the conditions to obtain the maximum gains and losses of energy, depending on the goal of the maneuver. They also showed that gains and losses of energy have some peculiarities and need to be addressed with some differences in the approach used to solve the problem, so the present paper considers only the situation where a gain of energy is desired.

Following the lines presented in references $[1,4]$, the present research focuses on calculating the energy variation in a maneuver where the application of the impulse occurs in a free point inside the sphere of influence of the secondary celestial body, as done in [4]. Therefore, the present paper makes several generalizations of those previous references, as explained next. The production of general maps for the energy gains given by the maneuver, as a function of all the important parameters involved, gives more information compared to the previous papers, which are concentrated in finding the maximum gains of energy. Maximum gain of energy is of course a very important aspect, but it is not the only point that deserves to be studied. The geometric parameters, like the angle of approach and periapsis distance, are related to the whole trajectory and the goals of the mission. So, it is interesting to choose them considering several factors, not only the maximization of the energy variation. In that way, maps of energy variations can help mission designers to attend the several constraints of a mission, by given sets of solutions for any combination of arrival parameters, including the values for the point of application and direction of the impulse that maximizes the energy variation. Those sets of solutions can also help to correct errors that may occur in the trajectory injection and/or intermediate maneuvers. Those steps may generate trajectories arriving to the secondary body which may be different from the expected ones. Those maps also show the regions of captures and escapes of the spacecraft, so giving different options for a given magnitude of the impulse available, which are controlled by the direction of the impulse and the location of the application point. It is also important to note that several details of each solution are shown in plots and tables, so giving all the information required to plan a mission in advance or to minimize problems due to errors in the arrival trajectory. The solutions presented here are obtained using an accurate model, the regularized restricted three-body problem, which is an important factor when considering the Earth-Moon system. This particular system has a high mass ratio and so introduces large errors in the results obtained with the "patched-conics" model. The errors increase because the spacecraft spends too much time in intermediate regions that are not highly dominated by one of the primaries. Those errors are eliminated by adopting our numerical approach that takes into account the whole three-body system. Simulations are made for two different systems of primaries, Earth-Moon and Sun-Jupiter, to show the importance of the mass parameter in those maneuvers. It should be emphasized, at this point, that a global optimization of the whole trajectory is a mandatory and important step in this type of problem but, as done in references $[1,2,4]$, an analyses of the powered Swing-By part of the mission can give several results that are useful when making the global planning, as shown in the next parts of the present paper. It is even possible to compensate errors in the approach trajectory. In other words, the results shown here give several "a priori" information that can guide a global optimization of the mission.

\section{Literature review}

There are many publications in topics related to SwingBys $[5,6]$. The Voyager mission was one of the first and most famous mission using this concept $[7,8]$. Byrnes and D' Amario [9] studied the Galileo mission that was sent to Jupiter. Multiple Swing-By trajectories using the Moon were considered in reference [10]. The Ulysses mission used this technique to observe the poles of the Sun, by making a three-dimensional Swing-By with Jupiter [11]. Swing-By trajectories around Jupiter and passing near the Earth were also mapped [12,13], as well as around the Moon [14]. Sukhanov [15] studied maneuvers of this type in the inner planets of the solar system, before going to the Sun. Casalino et. al. [16] studied Swing-By maneuvers to maximize the energy of satellites. Longuski and Strange [17] developed graphical methods to design Swing-By trajectories. McConaghy et al. [18] combined low thrust with Swing-By maneuvers. The Moons of Jupiter have been considered for this type of mission, like making a trip to Europa [19] or to make close approaches with all the Galilean Moons [20,21]. Missions to Pluto can also benefit from this concept [22]. Trajectories to Mars using a passage by Venus are considered in Hollister 
and Prussing [23] and Striepe and Braun [24]. Another application is described in Muhonen et al. [25]. An analytical study was made by Broucke [26]. Araújo et al. [27] used the effect of Swing-By of single and multiple encounters between asteroids. Swing-By maneuvers combined with low thrust were considered in Okutsu et al. [28] and Santos et al. [29]. Recent examples of missions using Swing-Bys are Messenger and BepiColombo, both of them having the planet Mercury as a target (see McNutt et al. [30,31], Grard [32] and Jehn et al. [33]). There are also studies considering clouds of particles, instead of a single particle, as shown in references [34-36].

\section{System dynamics}

The dynamical system used in the present research is the restricted three-body problem [37-41] combined with the regularization of Lemaitre [38]. This technique is used to increase the accuracy of the numerical integrations when the spacecraft is passing near the primaries. The system consists of three bodies: $M_{1}$, the primary body; $M_{2}$, the secondary body that is in a circular orbit around $M_{1}$; and $M_{3}$, a spacecraft with a negligible mass that makes a Swing-By with $M_{2}$. The equations of motion of the restricted three-body problem using the rotating system of reference and the canonical system of units [38] are given by:

$$
\begin{aligned}
& \ddot{x}-2 \dot{y}=\frac{\partial \Omega}{\partial x}, \\
& \ddot{y}+2 \dot{x}=\frac{\partial \Omega}{\partial y}, \\
& \Omega=\frac{1}{2}\left(x^{2}+y^{2}\right)+\frac{1-\mu}{r_{1}}+\frac{\mu}{r_{2}} .
\end{aligned}
$$

In those equations, $x$ and $y$ represent the position of the spacecraft, $\mu$ the mass of the smaller body in canonical units, $r_{1}$ the distance between $M_{1}$ and $M_{3}$ and $r_{2}$ the distance between $M_{2}$ and $M_{3}$. Those equations of motion depend on the potential $\Omega$, which depends on $r_{1}$ and $r_{2}$ in the denominator. When $M_{3}$ approaches one of the primaries, $r_{1}$ or $r_{2}$ becomes small, and this fact may affect the accuracy of the numerical integration. The purpose of the regularization technique is to eliminate these singularities, by using the technique of replacing variables. Regularization can be classified as local, when the singularities are removed one at a time, or global, when the two singularities are eliminated simultaneously. Several transformations are available in the literature, like Thiele-Burrau, Lemaître and Birkhoff [38]. In this work the global regularization proposed by Lemaître is used, which proofed to be efficient in this type of maneuver [12-14]. To perform the required transformation it is necessary to introduce a complex variable $q=q_{1}+q_{2} i$, where $\mathrm{i}$ is the imaginary unit, as follows.

$$
\begin{aligned}
& q_{1}=x+\frac{1}{2}-\mu \\
& q_{2}=y
\end{aligned}
$$

This transformation defines a new plane for the reference system $\left(q_{1}-q_{2}\right)$, which has its center exactly at the average distance between the bodies $M_{1}$ and $M_{2}$. This is a first change of variables, but it is necessary to make a second change of variables. For the Lemaitre regularization, this second transformation of variables for the position is done using the function $f(\omega)$ defined by Eq. (6). In this equation $\omega=\omega_{1}+i \omega_{2}$ is the new complex variable used to represent the position of the spacecraft. Equation (7) defines the transformation of time, so defining the variable $\tau$, which is the regularized time. The symbol $f^{\prime}(\omega)$ means the derivative of $f(\omega)$ with respect to the regularized time.

$$
\begin{gathered}
q=f(\omega)=\frac{1}{4}\left(\omega^{2}+\frac{1}{\omega^{2}}\right) \\
\frac{\partial t}{\partial \tau}=\left|f^{\prime}(\omega)\right|^{2}=\frac{\left|\omega^{4}-1\right|^{2}}{4|\omega|^{6}}
\end{gathered}
$$

The equation of motion for the particle with negligible mass, under the model given by the restricted three-body problem, in the complex form (variables $q_{1}, q_{2}$ ), is given by Eq. (8). Over dots mean derivative with respect to the physical original time and $U$ the potential.

$\ddot{q}+2 i \dot{q}=\nabla U(q)$.

This same equation of motion of the spacecraft, in the new variables, becomes

$\omega^{\prime \prime}+2 i\left|f^{\prime}(\omega)\right| \omega^{\prime}=\nabla \Omega^{*}$.

In Eq. (9) $\nabla \Omega^{*}$ represents $\frac{\partial \Omega^{*}}{\partial \omega_{1}}+\frac{\partial \Omega^{*}}{\partial \omega_{2}}, \Omega^{*}=$ $\left(\Omega-\frac{c_{1}}{2}\right)\left|f^{\prime}(\omega)\right|^{2}$, with $C_{1}-\mu(1-\mu)-2 J$ and $\mathrm{J}$ the Jacobi integral; $\omega^{\prime}$ and $\omega^{\prime \prime}$ denote first and second derivatives of $\omega$ with respect to the regularized time $\tau$. 




Fig. 1 The pure gravity Swing-By maneuver. Prado [1]

Next, it is important to look into some detail at the pure gravity Swing-By maneuver. This maneuver needs three independent parameters to be uniquely identified. In the present research, the following set of parameters is used [1]:

(i) $V_{\infty-}$, the magnitude of the velocity of the spacecraft when approaching the secondary body;

(ii) $r_{p}$, the periapsis distance of the hyperbolic orbit of the spacecraft around $M_{2}$;

(iii) $\psi$, the angle between the periapsis line and the line $M_{1}-M_{2}$.

Figure 1 shows some details of the pure gravity Swing-By maneuver, as well as the most important variables used in the present paper. Since this third body has a negligible mass, it does not interfere in the motion of the two primaries. They travel in circular orbits around their center of mass, in the version of the problem considered here. The spacecraft arrives at the sphere of influence [3] of $M_{2}$ at the point $A$; travels to the periapsis of its trajectory around $M_{2}$ (point marked $P$ ); and then leaves the sphere of influence of $M_{2}$ at the point $B$. At the points $A$ and $B$, the influence of $M_{2}$ is neglected and the two-body energy spacecraft- $M_{1}$ is assumed to be constant after $B$ and before $A$ (the orbits are assumed to be Keplerian). Two of the initial conditions are marked in Fig. 1: the periapsis distance $r_{p}$ (from the point $P$ to the center of $M_{2}$ ) and the angle of approach $\psi$, counted from the $M_{1}-M_{2}$ line in the counterclockwise sense. The distances are not in scale, to provide an easier visualization of the geometry of the maneuver. Note that only planar motions are allowed for the spacecraft in the present research.

Remember that this maneuver changes the velocity, energy and angular momentum of the spacecraft in its orbit with respect to $M_{1}$, due to the close passage by $M_{2}$. It is assumed that the orbits are Keplerian before the point $A$ and after the point $B$. There are equations to measure those changes in the literature, based in the "patched-conics" approach [26]. This approximation divides the total maneuver into three parts, all of them governed by the Keplerian celestial mechanics. The first part studies the motion of the spacecraft around $\mathrm{M}_{1}$ before the close encounter, neglecting the effects of $M_{2}$. When the spacecraft $\left(M_{3}\right)$ enters the sphere of influence of $M_{2}$, the effects of $M_{1}$ are neglected and another Keplerian system is formed, now with the bodies $M_{3}-M_{2}$. After that, the spacecraft leaves $M_{2}$ and goes back to another orbit around $M_{1}$. The Keplerian system $M_{3}-M_{1}$ is formed again, but the new orbit is different from the first one. The main goal of this maneuver is to modify these two Keplerian orbits around $M_{1}$. The equations used for those calculations [26] are given by Eqs. (10-13). Note that those equations are just a guide to the most suitable geometry for a desired maneuver. The results shown in the present paper are based in numerical integrations of the more accurate restricted three-body regularized problem, as already explained before.

$\delta=\sin ^{-1}\left[\left(1+\frac{r_{p} V_{\infty-}^{2}}{\mu_{2}}\right)^{-1}\right]$

$\Delta V=2 V_{\infty-} \sin \delta$

$\Delta E^{\prime}=-2 V_{2} V_{\infty-} \sin \delta \sin \psi$

$\Delta C=-2 V_{2} V_{\infty-} \sin \delta \sin \psi$

In those equations $\delta$ is the curvature of the trajectory of the spacecraft (Fig. 1), $V_{2}$ is the velocity of $M_{2}$ around the center of mass of the system $M_{1}-M_{2}, \Delta V$ is the increment of velocity obtained from the close approach, $\mu_{2}$ is the gravitational parameter of the body $M_{2}, \Delta E^{\prime}$ is the energy variation, and $\Delta C$ is the angular momentum variation. Equations (12) and (13) are the same because the angular velocity of the system is one. The general relation is $\Delta E^{\prime}=\omega_{A} \Delta C$, with $\omega_{A}$ the angular velocity of the rotating reference frame [26].

\section{Results}

The results show the variation of energy of the spacecraft under different parameters of the maneuver. The direction, magnitude and location of the impulse are varied. The main idea is to generate maps showing the 


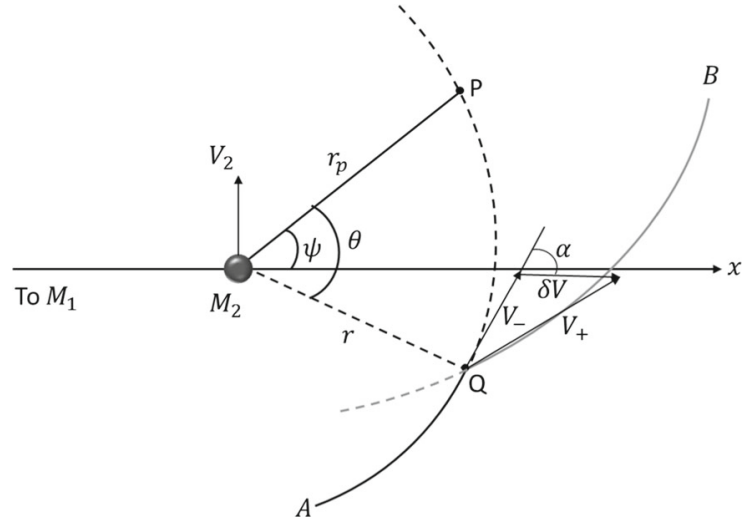

Fig. 2 Geometry of the powered Swing-By maneuver

variation of energy as a function of those parameters, but, from the results obtained, it is also possible to verify the parameters that give maximum energy variation for the maneuver, if this is the goal of the mission. It is important to remember that the geometric parameters of the maneuver (angle and velocity of approach and periapsis distance) are given based in the unpowered maneuver, because these are the parameters used to design the incoming trajectories. The real values are modified by the impulsive maneuver, so modifying the target values that give maximum variations of energy. Figure 2 describes in detail the powered maneuver considered in the present paper. The position $\boldsymbol{r}=(x, y)$ of the point where the impulse $\delta \boldsymbol{V}$ is applied is specified by the true anomaly of this point with respect to the hyperbolic orbit of $M_{3}$ around $M_{2}$, designated by $\theta$. It is assumed that $\theta$ is positive in the counterclockwise sense, measured from the periapsis vector $\boldsymbol{r}_{\boldsymbol{p}}=\left(x_{p}, y_{p}\right)$. The darker black line represents the first orbit, before the impulse is applied. The gray line represents the second orbit, after the impulse. The dashed parts of the trajectories are the continuations of the two orbits (with and without the impulse), in the parts that do not really exist.

As shown in Fig. 2, the position where the impulse is applied is a function of $\theta$. This angle can be obtained from the scalar product of the vectors $\boldsymbol{r}_{\boldsymbol{p}}$ and $\boldsymbol{r}$, so it is possible to get it from

$\theta=\cos ^{-1}\left(\frac{x_{p} x+y_{p} y}{r_{p} r}\right)$.

The algorithm for the numerical solution of the problem is shown below. (i) The study starts with the spacecraft placed at the point $P$. This is done by getting the initial conditions from the three variables that uniquely define a Swing-By trajectory: $V_{\infty_{-}}$, the magnitude of the velocity of the spacecraft when it is approaching the secondary body, which is transformed in $V_{p}$, the magnitude of the velocity of the spacecraft when passing by the periapsis (this transformation is made by assuming a Keplerian orbit around $M_{2}$ for this approach); $r_{p}$, the periapsis distance; and $\psi$, the angle between the line connecting the two primaries and the line of the periapsis;

(ii) From there, a numerical integration is performed until the spacecraft reaches the point $Q$, shown in Fig. 2, where the impulse is applied. This point is defined by the angle $\theta$;

(iii) From this point a numerical integration is made in reverse time [42], for the first orbit without the impulse, until a point $\mathrm{A}$, far from $M_{2}$, is reached. At point $\mathrm{A}$ the values of the energy $\left(E_{-}\right)$, velocity and angular momentum $\left(C_{-}\right)$of the spacecraft before the maneuver are obtained, using equations from the two-body dynamics $\left(M_{1}-M_{3}\right)$, which are assumed to be valid at the point $\mathrm{A}$ :

$$
\begin{aligned}
& E_{-}=\frac{(x+\dot{y})^{2}+(\dot{x}-y)^{2}}{2}-\frac{(1-\mu)}{r_{1}} \\
& C_{-}=x^{2}+y^{2}+x \dot{y}-y \dot{x}
\end{aligned}
$$

(iv) The impulse $\delta V$ is applied at the point $Q$, forming an angle $\alpha$ with the direction of the motion of the spacecraft. The magnitude and direction of the impulse are free parameters, used to reach the desired goals of the maneuver;

(v) An integration forward in time gives the new orbit after the impulse. The integration starts just after the impulse is applied and goes to a point B, distant from $M_{2}$. At this point it is possible to obtain the energy $\left(E_{+}\right)$, velocity and angular momentum $\left(C_{+}\right)$of the spacecraft after the complete maneuver;

$$
\begin{aligned}
& E_{+}=\frac{(x+\dot{y})^{2}+(\dot{x}-y)^{2}}{2}-\frac{1-\mu}{r_{1}} \\
& C_{+}=x^{2}+y^{2}+x \dot{y}-y \dot{x}
\end{aligned}
$$

(vi) Finally, the variations are calculated. In particular the energy variation, the most important parameter in the present paper, is obtained as a function of the magnitude of the impulse $(\delta V)$, its direction $\alpha$ 
and the angle $\theta$, which defines the location of the application of the impulse. This quantity can then be written in the form shown in Eq. (19).

$\Delta E=f(\delta V, \alpha, \theta)$

Therefore, the problem is studied considering two sets of initial conditions. The first one is to specify the gravity maneuver, using the variable $r_{p}, \psi, V_{\infty}$. The second one defines the impulse, with the variables shown in Eq. 19. The position and velocity of the spacecraft when located in $Q$ are obtained by making numerical integrations of the equations of motion given by the restricted three-body problem, which is more accurate than the "patched-conics" model used before in the literature [1,4]. The integration has an initial point at $P$ and goes up to the position described by the true anomaly $\theta$. The integration is made using negative times when $\theta$ is negative and in positive times when $\theta$ is positive. The variation of energy is obtained from the difference of the energy of the orbits before and after the passage by the celestial body. Two systems are simulated: Earth-Moon-spacecraft and SunJupiter-spacecraft, with the goal of investigating the effects of the mass parameter of the system in the results shown here.

The results of the simulations focus on the energy variations as a function of $\alpha$ and $\theta$ for different values of $\psi, \delta V$ and $r_{p}$. For all the plots, the horizontal axis represents the variation of $\alpha$ and the vertical axis represents the variation of $\theta$, both in degrees. The variables related to some particular important cases are shown in “Appendices 1 and 2". They show the maximum energy variations $\left(\Delta E_{\max }\right)$ and its corresponding data, as the true anomaly of the point where the impulse is applied $(\theta)$, the angle between the velocity vector of the spacecraft and the direction of the impulse $(\alpha)$, the deflection angle $(\zeta)$, the escape velocity $\left(V_{\infty+}\right)$ and $R$, which is the distance between the spacecraft and the secondary body at the instant that the impulse is applied. Different values for $r_{p}$ are used to study the importance of this variable in this problem in some more detail. Results are first shown for the Earth-Moon-spacecraft system and then for the Sun-Jupiter-spacecraft system. The notation used here indicates the radius of the Moon by $R_{m}$, the radius of Jupiter by $R_{j}$ and canonical units by c.u. A lower limit for the periapsis distance is not used here, like done in reference [4], because the goal is to map all types of occurrences, including collisions and escapes. But a practical limit equal to the radius of the secondary body exists, since the collisions with the secondary body are indicated.

\subsection{Earth-Moon system}

The first results are presented for the Earth-Moon system, in Fig. 3. It shows the energy variations for $r_{p}=$ $1.02 R_{m}, \psi=270^{\circ}$ and $\delta V=0.1,0.3,0.5,1.0,1.5$ and $2.0 \mathrm{~km} / \mathrm{s}$. This value for the angle of approach is used because the idea is to study a situation where the energy variation is positive and $\psi=270^{\circ}$ is the case of maximum gains of energy for the unpowered maneuver. The increase of the magnitude of the impulse helps to give more energy to the spacecraft. This fact can be seen from the scales of the color codes. The upper limit increases very fast with the magnitude of the impulse. This is expected, since a larger impulse can give more energy to the system. Even considering that, the results shown here can quantify this increase, which is not easy to estimate due to the complex dynamics involved. Another point visible in Fig. 3 is that the regions of minimum energy are concentrated in the lateral borders of the figures. It means angles $\alpha$ near $\pm 180^{\circ}$, which corresponds to apply the impulse in the direction opposite to the motion of the spacecraft. This fact can be explained because a retrograde impulse removes energy from the spacecraft. Figure 3 also shows that the regions of maximum variations of energy are concentrated near the middle of the figures, with shifts to the left, in the regions near $\alpha=-20^{\circ}$, and to the top, in the regions near $\theta=50^{\circ}$. Those results are in agreement with references [1] and [4]. These locations can be explained by two facts: (i) an impulse with negative angles makes the spacecraft to move closer to the body, so getting more energy from the gravity part of the maneuver, which helps to increase the energy in a geometry with $\psi=270^{\circ}$; (ii) positive values for the locations of the impulse make the maneuver to be performed after the close approach, so getting the gains in energy from the maneuver before modifying the trajectory of the spacecraft. It is also noted that the regions where the spacecraft is captured by the Moon are near the borders of the plots (the blank regions in the plots), with the impulse applied in the retrograde direction. Those are also the regions where the minimum energy 

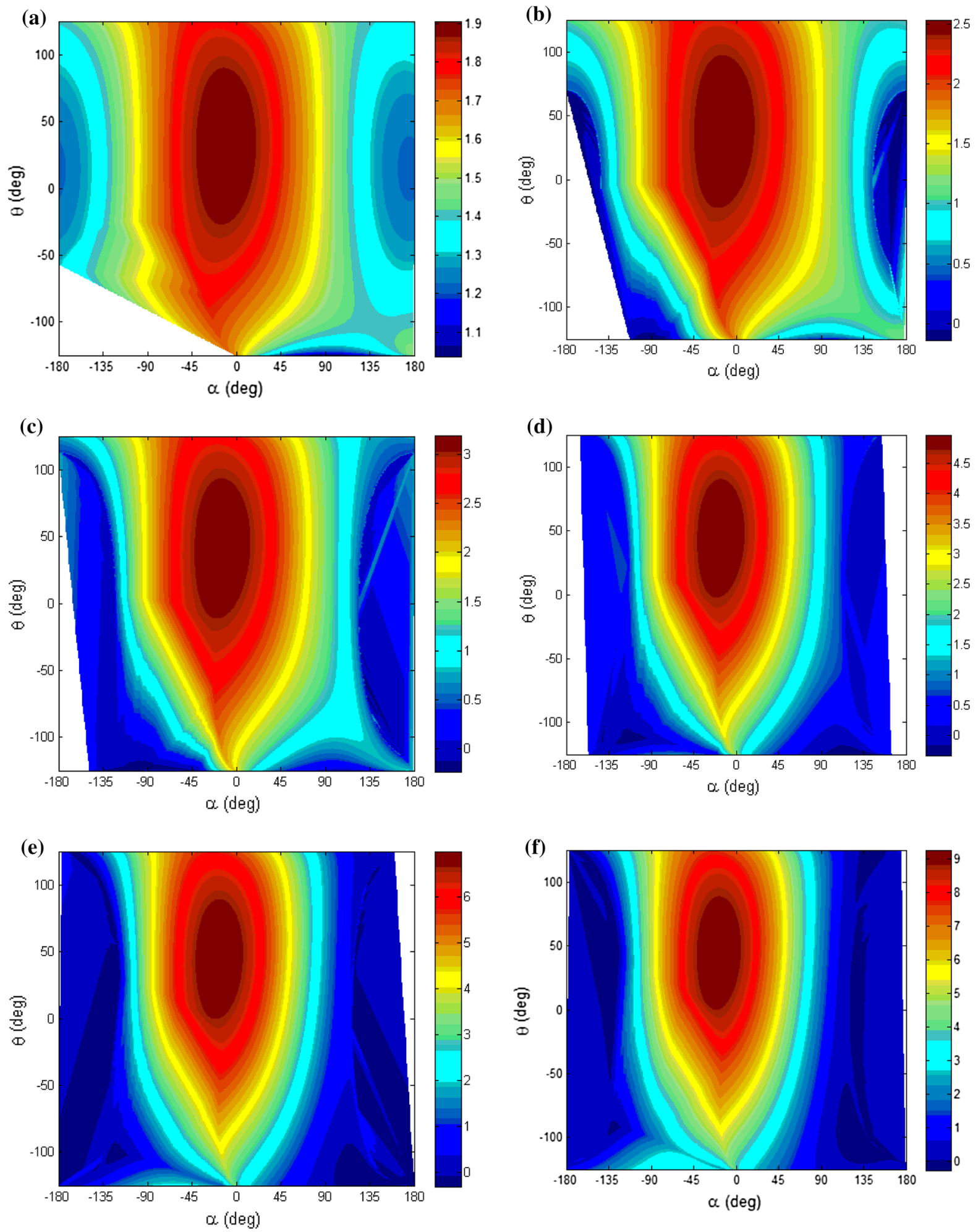

Fig. 3 Energy variation in $\mathrm{km}^{2} / \mathrm{s}^{2}$, for $r_{p}=1.02 R_{m}, \psi=270^{\circ}$ and $\delta V$ from 0.1 to $2.0 \mathrm{~km} / \mathrm{s}, \mathbf{a} \delta V=0.1 \mathrm{~km} / \mathrm{s}, \mathbf{b} \delta V=0.3 \mathrm{~km} / \mathrm{s}, \mathbf{c}$ $\delta V=0.5 \mathrm{~km} / \mathrm{s}, \mathbf{d} \delta V=1.0 \mathrm{~km} / \mathrm{s}, \mathbf{e} \delta V=1.5 \mathrm{~km} / \mathrm{s}, \mathbf{f} \delta V=2.0 \mathrm{~km} / \mathrm{s}$ 


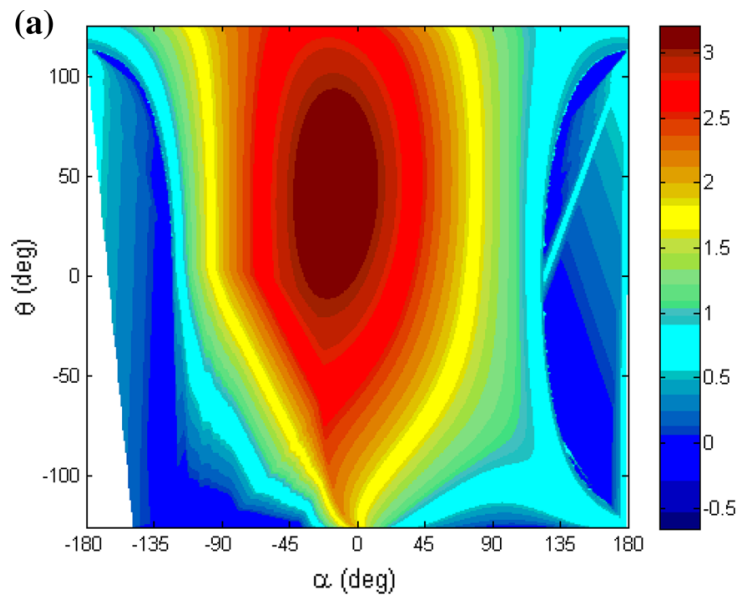

(c)

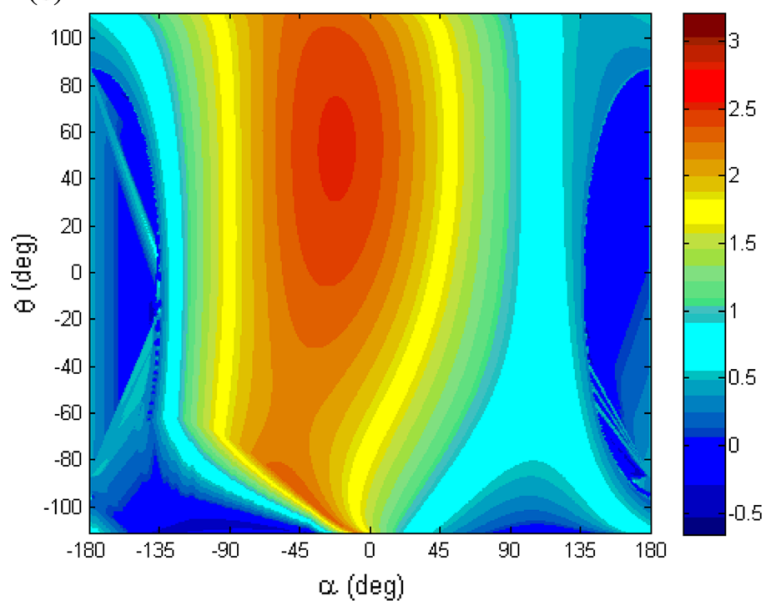

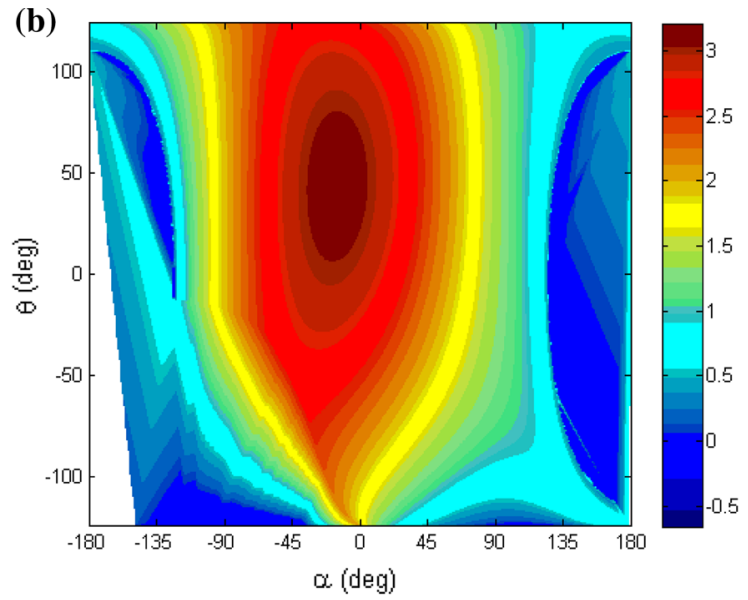

(d)

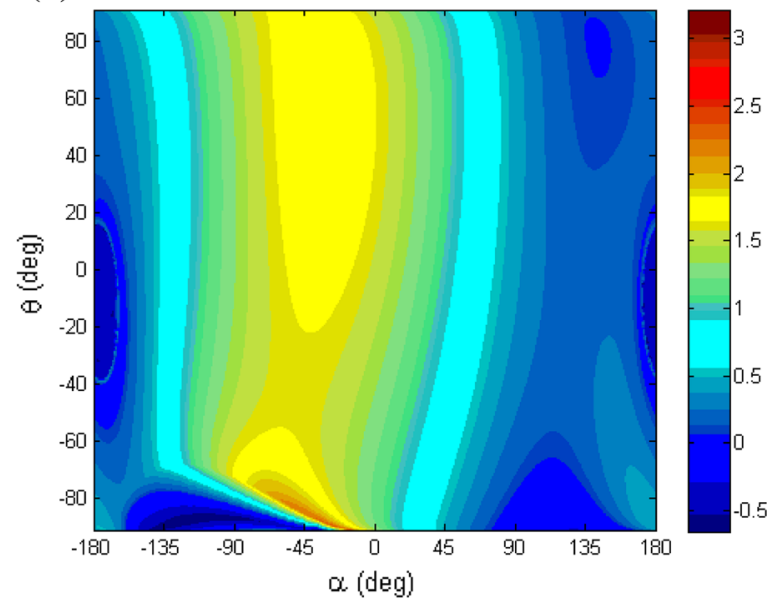

Fig. 4 Energy variation in $\mathrm{km}^{2} / \mathrm{s}^{2}$, for $\psi=270^{\circ}, \delta V=0.5 \mathrm{~km} / \mathrm{s}$ and different $r_{p}$ a $r_{p}=1.02 R_{m}, \mathbf{b} r_{p}=1.1 R_{m}, \mathbf{c} r_{p}=2.0 R_{m}, \mathbf{d}$ $r_{p}=5.0 R_{m}$

variations are concentrated. The reason is that retrograde impulses remove energy from the spacecraft.

Figure 4 shows the energy variation for $\psi=270^{\circ}$, $\delta V=0.5 \mathrm{~km} / \mathrm{s}$ and different values for the radius of the periapsis $\left(r_{p}\right)$. Note that the energy variations are in the same range. The minimum variations of energy are located in the lateral border of the plots, for the reasons already explained. The maximum energy variations are located near the center of the plots, with the same shifts already observed and explained. In this case, the increasing of the periapsis distance reduces the gains of energy, as expected.

The effects of the angle of approach $(\psi)$ in the maneuver are visible from Fig. 5. It presents the energy variation for $r_{p}=1.02 R_{m}, \delta V=0.5$ and different values for $\psi$. Because the goal of the maneu- ver is to get gains in energy, the interval $180^{\circ}<$ $\psi<360^{\circ}$ is used, which is the region of positive gains of energy. The results confirm the predictions based in the "patched-conics" approach, and maneuvers with $\psi=270^{\circ}$ give the maximum gains of energy. It is also noted that the values of the location of the impulses change with the angle of approach. The angle $\theta$ decreases with the value of $\psi$, going from near $75^{\circ}$, when $\psi=225^{\circ}$, to near $10^{\circ}$, when $\psi=315^{\circ}$. The angle that represents the direction of the impulse increases with the angle of approach, going from near $-30^{\circ}$, when $\psi=225^{\circ}$, to near $-5^{\circ}$, when $\psi=315^{\circ}$.

The blank regions in all the figures indicate collisions or captures of the spacecraft with respect to the secondary body, as explained before. 

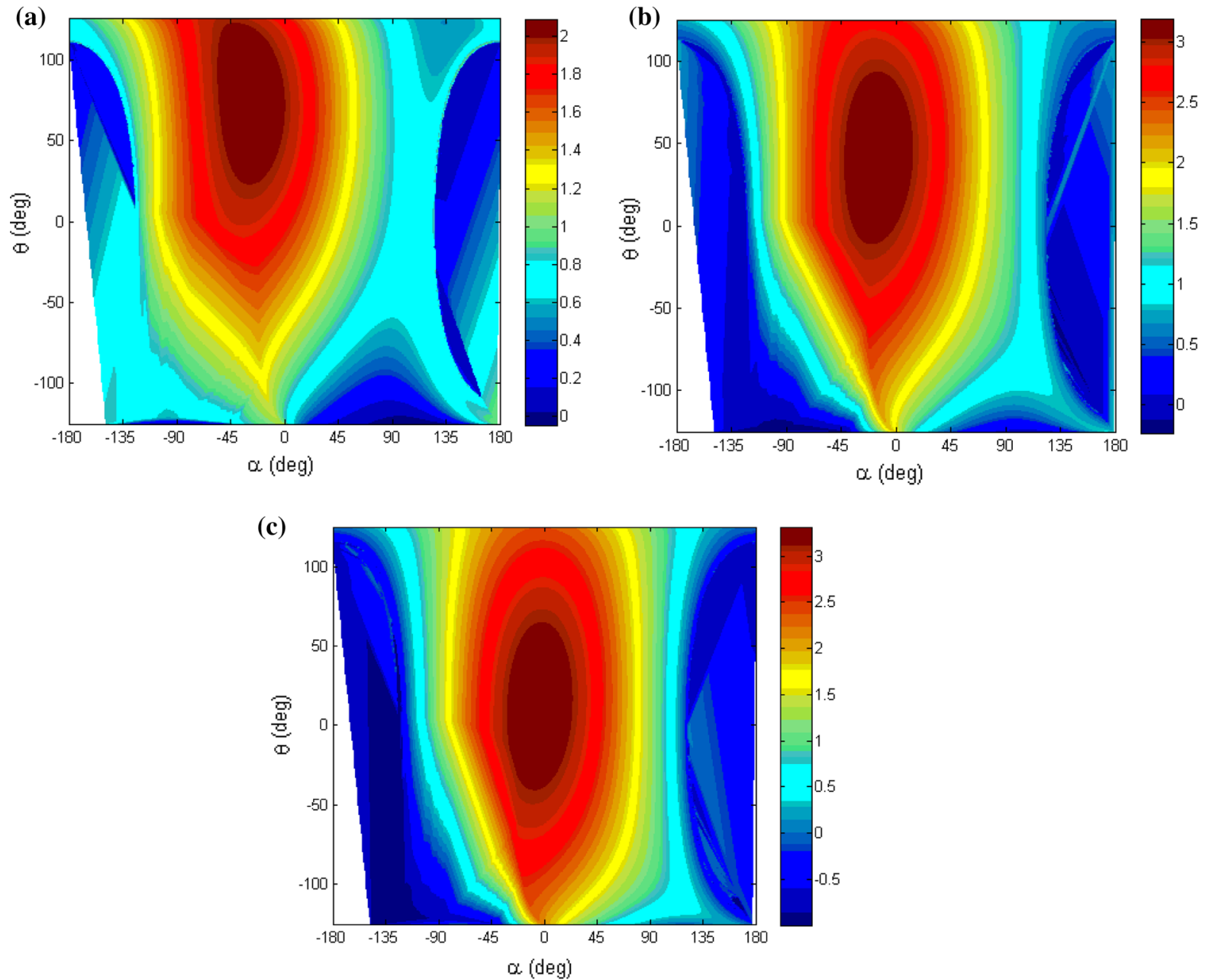

Fig. 5 Energy variation in $\mathrm{km}^{2} / \mathrm{s}^{2}$, for $r_{p}=1.02 R_{m}, \delta V=0.5$ and different values of $\psi \mathbf{a} \psi=225^{\circ}, \mathbf{b} \psi=270^{\circ}, \mathbf{c} \psi=315^{\circ}$

The maximum energy variations are summarized in Figs. 6 and 7, for $r_{p}=1.02$ and $2.0 R_{m}$, respectively. Figure (a) shows the angle of approach $(\psi)$ versus the angle that defines the direction of the impulse $(\alpha)$. Figure (b) shows the angle of approach $(\psi)$ versus the angle that defines the application point of the impulse $(\theta)$. Figure (c) shows the angle of approach $(\psi)$ versus the maximum energy variation $\left(\Delta E_{\max }\right)$. All the cases considered a magnitude of impulse from $\delta V=0.1$ to $\delta V=4.0 \mathrm{~km} / \mathrm{s}$. As expected, the maneuvers with maximum energy variations try to get as most energy as possible from the impulsive and the gravity part of the maneuver. In Fig. 6a it is noted that, for $\psi$ in the range from $190^{\circ}$ to $225^{\circ}$, the maneuver tries to change the final angle of approach such that it gets as close as possible to $270^{\circ}$, the point of maximum gains of energy for the gravity part of the maneuver. It makes $\alpha$ to vary in a larger range, depending on the value of the magnitude of the impulse. It is noted a more uniform behavior after $\psi=225^{\circ}$. The best values started near $-30^{\circ}$, for $\psi=225^{\circ}$, and then increased almost linearly, reaching values near zero when $\psi=350^{\circ}$. For this situation, the modifications in the angle of approach are small, because it is already close to the optimal point, and then $\alpha$ assumes small values to obtain the maximum effects from the impulse itself. The lines representing the magnitude of the impulses are near each other, and they are parallel, showing that this parameter does not modify the general behavior of the effects. The same fact occurs for the location of the impulse, with a more uniform behavior in the region of positive gains of energy. The best values started between $50^{\circ}$ 

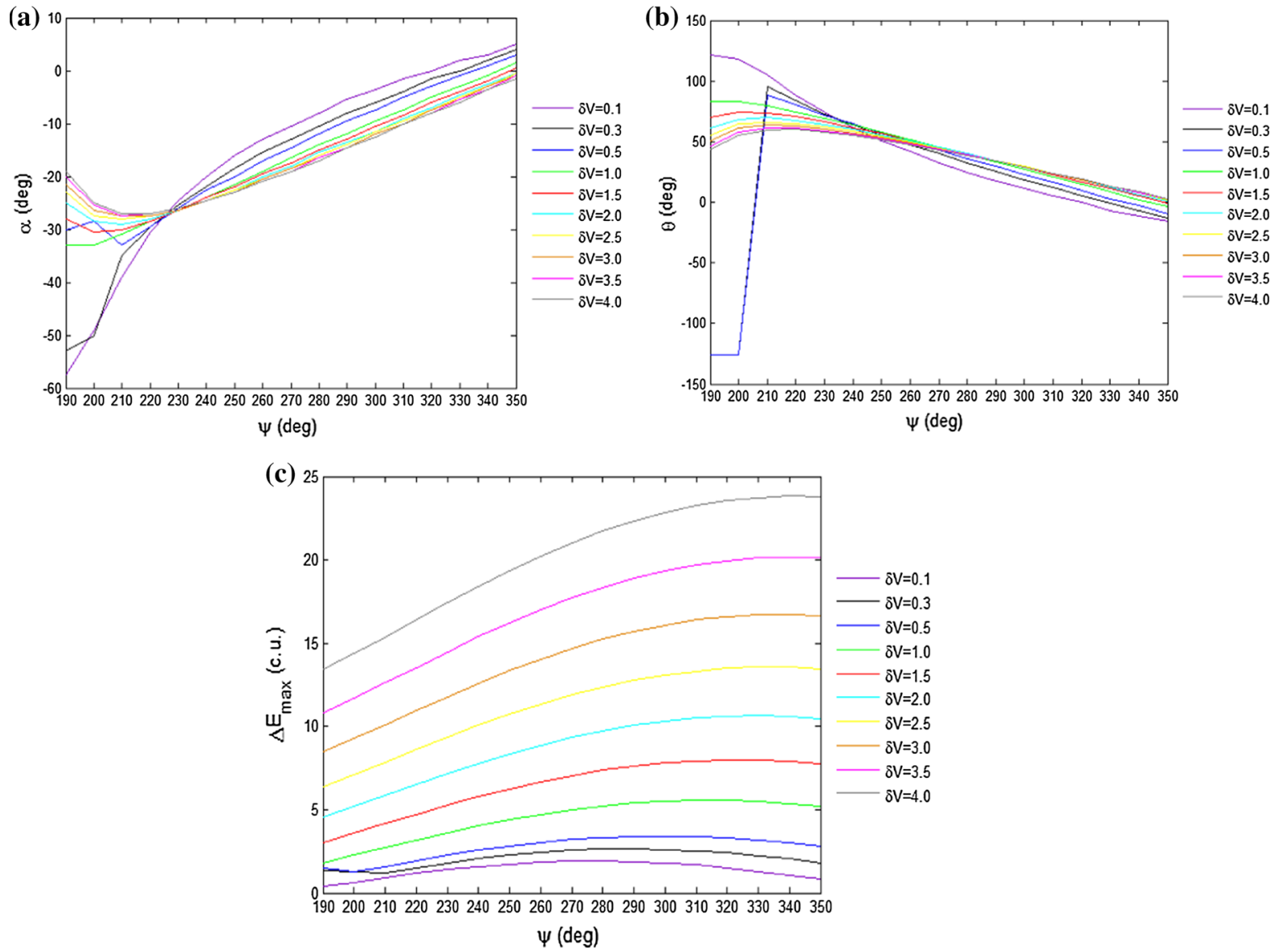

Fig. 6 a $\psi$ versus $\alpha$, $\mathbf{b} \psi$ versus $\theta$ and $\mathbf{c} \psi$ versus $\Delta E_{\max }$ for $1.02 \mathrm{R}_{m}$

and $70^{\circ}$, depending on the magnitude of the impulse, for $\psi=225^{\circ}$, and then it decreased almost linearly, reaching values near zero or negative when $\psi=350^{\circ}$. The lines representing the magnitude of the impulses are near each other and parallel, as in the previous case, showing that this parameter does not modify the general behavior of the effects. The variations of energy are very affected by the magnitude of the impulse. For smaller values, it follows closely the results predicted by the "patched-conics" approach, with a curve that is symmetric with respect to the line $\psi=270^{\circ}$, having a maximum at this point. The increase in the magnitudes of the impulse gives larger variations of energy, as expected and mentioned before, but it also changes the general behavior of the curves. The locations of the maximum are modified and go to $\psi=315^{\circ}$. Figure 5 is made for a larger distance of periapsis, so the effects of the gravity part of the Swing-By are reduced. It has more negative values for the location of the periapsis, because negative values allow more control over the trajectory to get the most energy possible from the gravity part of the maneuver. The impulse generates a trajectory with a smaller periapsis distance, which was not possible in the previous cases, because it was already very near to the surface of the Moon. The values of the direction of the impulse also go to larger negative values, to make the spacecraft to get closer to the Moon during the passage. This type of plots is new in the literature and can help to plan the whole mission. The plot shown in Fig. 6, letter c, shows the maximum variations of energy as a function of the angle of approach and the magnitude of the impulse. The information coming from this plot can be seen better by looking at horizontal and vertical lines. A vertical line represents a fixed angle of approach. From this line it is possible to get the minimum magnitude of the impulse to obtain a given 

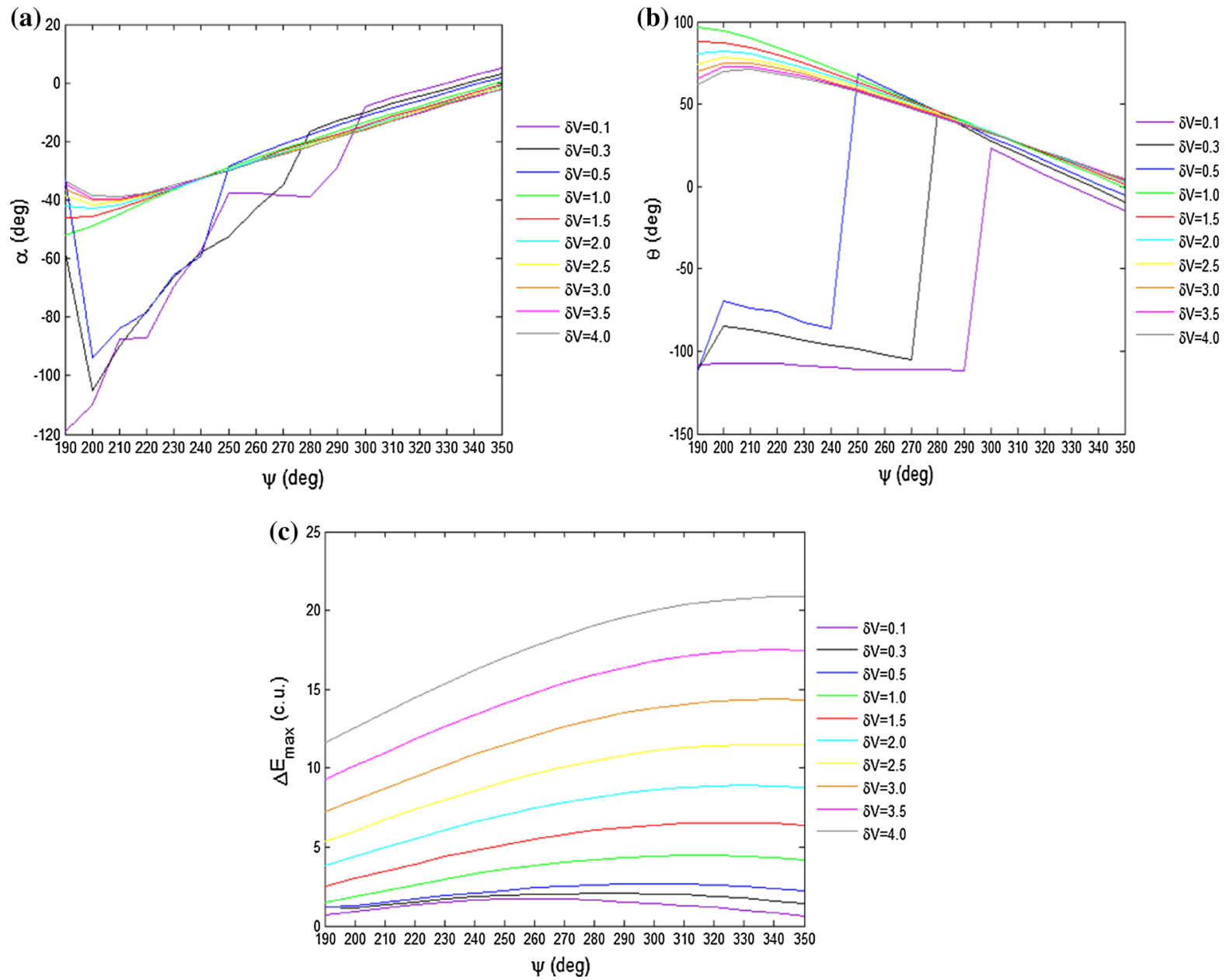

Fig. 7 a $\psi$ versus $\alpha, \mathbf{b} \psi$ versus $\theta$ and $\mathbf{c} \psi$ versus $\Delta E_{\max }$ for $2.0 R_{m}$

variation of energy, which is usually the requirement of a mission. It also gives the maximum variation of energy for a given magnitude of the impulse available to the spacecraft. The horizontal lines represent a fixed variation of energy. From those lines it is possible to see the value of the angle of approach that minimizes the magnitude of the impulse required. It is shown that it is not $270^{\circ}$, as one could expect in a first analysis. It happens because the angles of approach shown in the plots are referred to the unpowered maneuver, and the impulse changes the geometry of the system. Those lines also give the minimum magnitude of the impulse for a given angle of approach. This information can be combined with other constraints to choose the best geometry for the maneuver. After this choice is made, the plots shown in Fig. 6a, b give the solution of prob- lem, which are the point of application and the direction of the impulse that gives the maximum variation of energy.

It is also possible to use those results to correct errors in the arriving trajectory. If the arriving trajectory is not the expected one, it is possible to find the maximum variation of energy that can be obtained for the real value of the angle of approach. Therefore, the magnitude, direction and location of the impulse are found in Fig. 6. In this way, the mission designer can correct errors in the approach trajectory by making the best choice of the location, direction and magnitude of the impulse. The same arguments can be given for Fig. 7, which is similar to Fig. 6, just made for a different value of the periapsis distance. 
Table 1 Information about the maximum energy variation for $r_{p}=1.02 R_{m}$ in the Earth-Moon-spacecraft system

\begin{tabular}{|c|c|c|c|c|c|c|c|c|}
\hline$\delta V(\mathrm{~km} / \mathrm{s})$ & $\begin{array}{l}\Delta E_{\max }\left(\mathrm{km}^{2} / \mathrm{s}^{2}\right), \\
\text { for } \theta=0^{\circ}\end{array}$ & $\begin{array}{l}\alpha\left(^{\circ}\right) \\
\text { for } \theta=0^{\circ}\end{array}$ & $\Delta E_{\max }\left(\mathrm{km}^{2} / \mathrm{s}^{2}\right)$ & $\alpha\left(^{\circ}\right)$ & $\theta\left({ }^{\circ}\right)$ & $\zeta\left(^{\circ}\right)$ & $V_{\infty+}$ (c.u.) & $R$ (c.u.) \\
\hline \multicolumn{9}{|l|}{$\psi=225^{\circ}$} \\
\hline 0.1 & 1.2460 & -36.0 & 1.2853 & -27.5 & 80.5 & -9.8 & 1.3721 & 0.0089 \\
\hline 0.3 & 1.5492 & -35.5 & 1.6702 & -27.5 & 78.5 & -3.6 & 1.6295 & 0.0086 \\
\hline 0.5 & 1.9080 & -34.0 & 2.1086 & -27.5 & 76.0 & 0.8 & 1.8770 & 0.0082 \\
\hline 1.0 & 3.0378 & -30.5 & 3.4167 & -27.5 & 71.5 & 7.7 & 2.4651 & 0.0076 \\
\hline 1.5 & 4.4786 & -28.0 & 5.0076 & -27.5 & 68.5 & 11.9 & 3.0250 & 0.0073 \\
\hline 2.0 & 6.2069 & -24.0 & 6.8690 & -27.0 & 65.5 & 14.4 & 3.5760 & 0.0070 \\
\hline 2.5 & 8.2092 & -21.5 & 8.9946 & -27.0 & 63.5 & 16.3 & 4.1111 & 0.0068 \\
\hline 3.0 & 10.4806 & -20.0 & 11.3804 & -27.0 & 62.0 & 17.9 & 4.6365 & 0.0066 \\
\hline 3.5 & 13.0116 & -18.5 & 14.0238 & -27.0 & 60.5 & 19.0 & 5.1571 & 0.0065 \\
\hline 4.0 & 15.8039 & -17.5 & 16.9234 & -26.5 & 59.0 & 19.6 & 5.6807 & 0.0064 \\
\hline \multicolumn{9}{|l|}{$\psi=270^{\circ}$} \\
\hline 0.1 & 1.8997 & -12.0 & 1.9132 & -10.5 & 32.5 & -14.4 & 1.4230 & 0.0050 \\
\hline 0.3 & 2.4971 & -16.0 & 2.5544 & -13.0 & 40.0 & -9.4 & 1.7275 & 0.0053 \\
\hline 0.5 & 3.1057 & -18.0 & 3.2147 & -14.5 & 43.0 & -5.9 & 1.9991 & 0.0054 \\
\hline 1.0 & 4.7760 & -20.5 & 5.0150 & -16.5 & 45.5 & -0.2 & 2.6153 & 0.0056 \\
\hline 1.5 & 6.6987 & -21.0 & 7.0566 & -17.5 & 45.5 & 3.3 & 3.1894 & 0.0056 \\
\hline 2.0 & 8.8822 & -21.5 & 9.3477 & -18.0 & 45.0 & 5.8 & 3.7414 & 0.0055 \\
\hline 2.5 & 11.3260 & -21.5 & 11.8900 & -18.5 & 44.5 & 7.8 & 42.2776 & 0.0055 \\
\hline 3.0 & 14.0254 & -20.0 & 14.6838 & -18.5 & 44.0 & 9.1 & 4.8082 & 0.0055 \\
\hline 3.5 & 16.9739 & -18.5 & 17.7292 & -19.0 & 43.5 & 10.6 & 5.3265 & 0.0055 \\
\hline 4.0 & 20.1746 & -17.5 & 21.0259 & -19.0 & 43.0 & 11.4 & 5.8 & 0.0054 \\
\hline \multicolumn{9}{|l|}{$\psi=315^{\circ}$} \\
\hline 0.1 & 1.5980 & -1.0 & 1.5982 & -0.5 & 2.5 & 0.1 & 1.3303 & 0.0046 \\
\hline 0.3 & 2.4855 & -3.0 & 2.4891 & -2.5 & 9.1 & 3.8 & 1.6848 & 0.0046 \\
\hline 0.5 & 3.3385 & -4.5 & 3.3512 & -4.0 & 13.0 & 5.9 & 2.0 & 0.0047 \\
\hline 1.0 & 5.5172 & -7.5 & 5.5640 & -6.0 & 18.0 & 9.3 & 2.6609 & 0.0047 \\
\hline 1.5 & 7.8743 & -9.0 & 7.9587 & -7.5 & 19.6 & 11.6 & 3.2634 & 0.0047 \\
\hline 2.0 & 10.4545 & -10.0 & 10.5746 & -8.0 & 20.5 & 13.2 & 3.8344 & 0.0048 \\
\hline 2.5 & 13.2720 & -10.5 & 13.4255 & -8.5 & 21.0 & 14.6 & 4.3846 & 0.0048 \\
\hline 3.0 & 16.3328 & -11.0 & 16.5172 & -8.5 & 21.5 & 15.6 & 4.9233 & 0.0048 \\
\hline 3.5 & 19.6398 & -11.0 & 19.8531 & -9.0 & 21.5 & 16.6 & 5.4515 & 0.0048 \\
\hline 4.0 & 23.1941 & -11.5 & 23.4348 & -9.0 & 21.5 & 17.2 & 5.9752 & 0.0048 \\
\hline
\end{tabular}

Empirical equations were developed to estimate the maximum energy variations, from the independent variables $\psi$ and $\delta V$. The equations were obtained by making a curve fitting of the data available. Equation (20) refers to the data shown in Fig. 6c, for $r_{p}=1.02 R_{m}$, and their coefficients are described in "Appendix 3".

$\Delta E_{\max }=a_{1} \psi^{4}+b_{1} \psi^{3}+c_{1} \psi^{2}+d_{1} \psi+e_{1}$
Empirical Eq. (21) refers to the data shown in Fig. 7c, for $r_{p}=2.0 R_{m}$. The coefficients are described in "Appendix 3".

$\Delta E_{\max }=a_{2} \psi^{4}+b_{2} \psi^{3}+c_{2} \psi^{2}+d_{2} \psi+e_{2}$

The largest magnitude of $\Delta E_{\max }$ occurs for $r_{p}=$ $1.02, \delta V=4.0 \mathrm{~km} / \mathrm{s}$ and $\psi=350^{\circ}$. The value for 
Table 2 Information about the maximum energy variation with $r_{p}=1.1 R_{m}$ in the Earth-Moon-spacecraft system

\begin{tabular}{|c|c|c|c|c|c|c|c|c|}
\hline$\delta V(\mathrm{~km} / \mathrm{s})$ & $\begin{array}{l}\Delta E_{\max }\left(\mathrm{km}^{2} / \mathrm{s}^{2}\right) \\
\text { for } \theta=0^{\circ}\end{array}$ & $\alpha\left(^{\circ}\right)$ for $\theta=0^{\circ}$ & $\Delta \mathrm{E}_{\max }\left(\mathrm{km}^{2} / \mathrm{s}^{2}\right)$ & $\alpha\left(^{\circ}\right)$ & $\theta\left(^{\circ}\right)$ & $\zeta\left(^{\circ}\right)$ & $\mathrm{V}_{\infty+}$ (c.u.) & R(c.u.) \\
\hline \multicolumn{9}{|l|}{$\psi=225^{\circ}$} \\
\hline 0.1 & 1.2185 & -38.5 & 1.2586 & -29.5 & 84.5 & -12.5 & 1.3611 & 0.0105 \\
\hline 0.3 & 1.5102 & -37.5 & 1.6325 & -29.0 & 80.5 & -6.0 & 1.6106 & 0.0097 \\
\hline 0.5 & 1.8578 & -35.5 & 2.0597 & -29.0 & 78.0 & 1.4 & 1.8506 & 0.0092 \\
\hline 1.0 & 2.9591 & -31.5 & 3.3389 & -28.5 & 73.0 & 5.9 & 2.4299 & 0.0085 \\
\hline 1.5 & 4.3700 & -29.0 & 4.9001 & -28.5 & 69.5 & 10.2 & 2.9846 & 0.0080 \\
\hline 2.0 & 6.0702 & -27.5 & 6.7311 & 28.0 & 67.0 & 13.0 & 3.5280 & 0.0077 \\
\hline 2.5 & 8.0473 & -26.0 & 8.8257 & -28.0 & 65.0 & 15.1 & 4.0578 & 0.0075 \\
\hline 3.0 & 10.2934 & -25.5 & 11.1800 & -28.0 & 63.03 & 16.7 & 4.5820 & 0.0073 \\
\hline 3.5 & 12.8033 & -25.0 & 13.7915 & -28.0 & 61.5 & 17.9 & 5.1008 & 0.0071 \\
\hline 4.0 & 15.5739 & -24.5 & 16.6588 & -27.5 & 60.0 & 18.6 & 5.6215 & 0.0070 \\
\hline \multicolumn{9}{|l|}{$\psi=270^{\circ}$} \\
\hline 0.1 & 1.8551 & -13.0 & 1.8696 & -11.0 & 35.1 & -16.1 & 1.4183 & 0.0055 \\
\hline 0.3 & 2.4309 & -16.5 & 2.4903 & -13.5 & 41.5 & -11.0 & 1.7144 & 0.0058 \\
\hline 0.5 & 3.0221 & -19.0 & 3.1334 & -15.0 & 44.0 & -7.3 & 1.9797 & 0.0059 \\
\hline 1.0 & 4.6547 & -21.0 & 4.8951 & -17.0 & 46.5 & -1.2 & 2.5848 & 0.0060 \\
\hline 1.5 & 6.5422 & -21.5 & 6.9003 & -18.0 & 46.5 & 2.5 & 3.1518 & 0.0060 \\
\hline 2.0 & 8.6913 & -22.0 & 9.1555 & -18.5 & 46.0 & 5.2 & 3.6986 & 0.0060 \\
\hline 2.5 & 11.1007 & -22.0 & 11.6623 & -19.0 & 45.6 & 7.3 & 4.2306 & 0.0060 \\
\hline 3.0 & 13.7686 & -22.0 & 14.4206 & -19.0 & 45.0 & 8.7 & 4.7581 & 0.0060 \\
\hline 3.5 & 16.6932 & -22.0 & 17.4305 & -19.5 & 44.0 & 10.0 & 5.2771 & 0.0059 \\
\hline 4.0 & 19.8731 & -22.0 & 20.6917 & 19.5 & 43.5 & 11.0 & 5.7924 & 0.0059 \\
\hline \multicolumn{9}{|l|}{$\psi=315^{\circ}$} \\
\hline 0.1 & 1.5594 & -1.0 & 1.5596 & -1.0 & 2.5 & -2.3 & 1.3287 & 0.0049 \\
\hline 0.3 & 2.4191 & -3.5 & 2.4233 & -3.0 & 9.5 & 1.69 & 1.6736 & 0.0050 \\
\hline 0.5 & 3.2490 & -5.0 & 3.2628 & -4.5 & 13.6 & 4.0 & 1.9736 & 0.0050 \\
\hline 1.0 & 5.3789 & -8.0 & 5.4273 & -6.5 & 18.6 & 7.7 & 2.6326 & 0.0051 \\
\hline 1.5 & 7.6927 & -9.5 & 7.7785 & -7.5 & 20.0 & 10.1 & 3.2294 & 0.0051 \\
\hline 2.0 & 10.2319 & -10.0 & 10.3530 & -8.0 & 21.0 & 11.8 & 3.7954 & 0.0051 \\
\hline 2.5 & 13.0096 & -11.0 & 13.1635 & -8.5 & 21.5 & 13.3 & 4.3420 & 0.0052 \\
\hline 3.0 & 16.0312 & -11.0 & 16.2154 & -9.0 & 21.5 & 14.4 & 4.8770 & 0.0052 \\
\hline 3.5 & 19.2992 & -11.5 & 19.5117 & -9.0 & 21.5 & 15.3 & 5.4047 & 0.0052 \\
\hline 4.0 & 22.8151 & -11.5 & 23.0544 & -9.5 & 21.5 & 16.2 & 5.9238 & 0.0052 \\
\hline
\end{tabular}

$r_{p}$ was expected to be the minimum one, because the closer to the body is the maneuver, the stronger is the interaction between the bodies. The effects of the $\delta V$ were also as expected, since we concluded, from Fig. 3 , that the increase of the energy variation is directly proportional to the increase of the magnitude of the impulse. It is known that the energy obtained is a combination of the effects coming from the standard Swing-
By with the impulse applied. The fact that the trajectory is deviated and has a real angle of approach in the region of positive gains of energy also cooperates to make this maneuver the one with the largest maximum energy variation for the system studied. It explains the value obtained for the angle of approach, which is very far from $270^{\circ}$, the best value for the unpowered maneuver. 
Table 3 Information about the maximum energy variation for $r_{p}=2.0 R_{m}$ in the Earth-Moon-spacecraft system

\begin{tabular}{|c|c|c|c|c|c|c|c|c|}
\hline$\delta V(\mathrm{~km} / \mathrm{s})$ & $\begin{array}{l}\Delta E_{\max }\left(\mathrm{km}^{2} / \mathrm{s}^{2}\right) \\
\text { for } \theta=0^{\circ}\end{array}$ & $\alpha\left(^{\circ}\right)$ for $\theta=0^{\circ}$ & $\Delta E_{\max }\left(\mathrm{km}^{2} / \mathrm{s}^{2}\right)$ & $\alpha\left(^{\circ}\right)$ & $\theta\left(^{\circ}\right)$ & $\zeta\left(^{\circ}\right)$ & $V_{\infty+}$ (c.u.) & R(c.u.) \\
\hline \multicolumn{9}{|l|}{$\psi=225^{\circ}$} \\
\hline 0.1 & 0.9954 & -60.5 & 1.4341 & -77.0 & -108.0 & -14.8 & 1.2810 & 0.0650 \\
\hline 0.3 & 1.228 & -54.0 & 1.6140 & -74.5 & -91.0 & -10.3 & 1.3740 & 0.0262 \\
\hline 0.5 & 1.5118 & -49.0 & 1.8190 & -72.0 & -79.0 & -7.8 & 1.5104 & 0.0188 \\
\hline 1.0 & 2.4400 & -41.5 & 2.7930 & -38.5 & 82.0 & -9.6 & 2.1873 & 0.0202 \\
\hline 1.5 & 3.6644 & -38.0 & 4.1605 & -38.0 & 77.5 & -3.1 & 2.6931 & 0.0181 \\
\hline 2.0 & 5.1686 & -35.5 & 5.7921 & -37.5 & 74.5 & 1.3 & 3.1983 & 0.0170 \\
\hline 2.5 & 6.9428 & -34.5 & 7.6829 & -37.0 & 72.0 & 4.3 & 3.7051 & 0.0162 \\
\hline 3.0 & 8.9808 & -33.5 & 9.8302 & -36.5 & 70.0 & 6.5 & 4.2112 & 0.0156 \\
\hline 3.5 & 11.2788 & -32.5 & 12.2323 & -36.5 & 68.5 & 8.4 & 4.7099 & 0.0152 \\
\hline 4.0 & 13.8343 & -32.0 & 14.8879 & -36.5 & 67.0 & 9.9 & 5.2085 & 0.0148 \\
\hline \multicolumn{9}{|l|}{$\psi=270^{\circ}$} \\
\hline 0.1 & 1.4865 & -22.0 & 1.7037 & -38.5 & -111.0 & -11.4 & 1.2954 & 0.1020 \\
\hline 0.3 & 1.9317 & -24.5 & 2.0264 & -35.0 & -105.0 & -10.7 & 1.4771 & 0.0507 \\
\hline 0.5 & 2.4156 & -26.0 & 2.5346 & -21.0 & 53.0 & -17.8 & 1.8395 & 0.0121 \\
\hline 1.0 & 3.8121 & -27.0 & 4.0451 & -22.5 & 52.5 & -9.5 & 2.3754 & 0.0120 \\
\hline 1.5 & 5.4767 & -27.0 & 5.8103 & -23.0 & 51.5 & -4.2 & 2.8973 & 0.0119 \\
\hline 2.0 & 7.4051 & -27.5 & 7.8291 & -23.5 & 50.5 & -0.3479 & 3.4103 & 0.0117 \\
\hline 2.5 & 9.5934 & -27.5 & 10.1004 & -24.0 & 49.5 & 2.5407 & 3.9177 & 0.0116 \\
\hline 3.0 & 12.0391 & -27.5 & 12.6236 & -24.0 & 48.5 & 4.5 & 4.4258 & 0.0115 \\
\hline 3.5 & 14.7402 & -27.5 & 15.3983 & -24.5 & 48.0 & 6.5 & 4.9244 & 0.0114 \\
\hline 4.0 & 17.6955 & -27.5 & 18.4241 & -24.5 & 47.0 & 7.8 & 5.4276 & 0.0113 \\
\hline \multicolumn{9}{|l|}{$\psi=315^{\circ}$} \\
\hline 0.1 & 1.2447 & -3.5 & 1.2461 & -3.5 & 10.5 & -22.1 & 1.3213 & 0.0091 \\
\hline 0.3 & 1.9191 & -7.0 & 1.9288 & -6.0 & 16.5 & -16.9 & 1.5990 & 0.0092 \\
\hline 0.5 & 2.5975 & -8.5 & 2.6196 & -7.0 & 20.0 & -13.1 & 1.8521 & 0.0094 \\
\hline 1.0 & 4.4099 & -11.0 & 4.4667 & -9.0 & 22.5 & -7.1 & 2.4373 & 0.0095 \\
\hline 1.5 & 6.4418 & -12.5 & 6.5312 & -10.0 & 24.0 & -3.0 & 2.9861 & 0.0095 \\
\hline 2.0 & 8.7130 & -13.0 & 8.8316 & -10.5 & 24.0 & -0.4 & 3.5194 & 0.0095 \\
\hline 2.5 & 11.2292 & -13.5 & 11.3744 & -11.0 & 24.0 & 1.8 & 40.0411 & 0.0095 \\
\hline 3.0 & 13.9929 & -14.0 & 14.1626 & -11.5 & 24.0 & 3.6 & 4.5554 & 0.0095 \\
\hline 3.5 & 17.0053 & -14.0 & 17.1977 & -11.5 & 23.5 & 4.9 & 5.0675 & 0.0095 \\
\hline 4.0 & 20.2666 & -14.0 & 20.4806 & -12.0 & 23.5 & 6.0 & 5.5727 & 0.0095 \\
\hline
\end{tabular}

“Appendix 1", Tables 1, 2, 3 and 4, show the results for $r_{p}=1.02 R_{m}, 1.1 R_{m}, 2.0 R_{m}$ and $5.0 R_{m}$, respectively, concerning the maximum variation of energy. The second and third columns show the maximum variation of energy and $\alpha$ for $\theta=0^{\circ}$. From the fourth column forward it is presented by the information for the optimum value of $\theta$, to show the gains obtained by optimizing this variable, instead of keeping it fixed in zero; $\zeta$, which is the deflection angle between the velocity vector of the spacecraft before and after the maneuver; $V_{\infty+}$, the magnitude of the velocity of the spacecraft when it leaves the secondary body; and $R$, the distance between the spacecraft and $M_{2}$ at the moment that the impulse is applied. Those tables confirm the previous results, with some detailed information about the variables involved. It also shows another important 
Table 4 Information about the maximum energy variation for $r_{p}=5.0 R_{m}$ in the Earth-Moon-spacecraft system

\begin{tabular}{|c|c|c|c|c|c|c|c|c|}
\hline$\delta V(\mathrm{~km} / \mathrm{s})$ & $\begin{array}{l}\Delta E_{\max }\left(\mathrm{km}^{2} / \mathrm{s}^{2}\right), \\
\text { for } \theta=0^{\circ}\end{array}$ & $\alpha\left(^{\circ}\right)$ for $\theta=0^{\circ}$ & $\Delta E_{\max }\left(\mathrm{km}^{2} / \mathrm{s}^{2}\right)$ & $\alpha\left(^{\circ}\right)$ & $\theta\left(^{\circ}\right)$ & $\zeta\left(^{\circ}\right)$ & $V_{\infty+}$ (c.u.) & $R$ (c.u.) \\
\hline \multicolumn{9}{|l|}{$\psi=225^{\circ}$} \\
\hline 0.1 & 0.6555 & -75.0 & 1.1962 & -89.5 & -90.5 & -36.9 & 1.2519 & 0.1534 \\
\hline 0.3 & 0.8690 & -71.0 & 1.8604 & -56.5 & -87.5 & -13.3 & 1.4246 & 0.1002 \\
\hline 0.5 & 1.1192 & -65.5 & 2.0874 & -57.5 & -81.5 & -11.4 & 1.5589 & 0.0693 \\
\hline 1.0 & 1.9312 & -56.0 & 2.7810 & -66.0 & -66.5 & -5.6 & 1.8804 & 0.0413 \\
\hline 1.5 & 3.0150 & -51.5 & 3.6767 & -69.5 & -54.0 & -2.3 & 2.2550 & 0.0330 \\
\hline 2.0 & 4.3650 & -49.0 & 4.8176 & -70.5 & -46.5 & -0.7 & 2.6783 & 0.0296 \\
\hline 2.5 & 5.9759 & -47.5 & 6.4474 & -50.0 & 68.0 & -12.5 & 3.3858 & 0.0438 \\
\hline 3.0 & 7.8445 & -46.5 & 8.3946 & -49.5 & 66.5 & -9.2 & 3.8682 & 0.0422 \\
\hline 3.5 & 9.9687 & -45.5 & 10.5942 & -49.5 & 65.5 & -6.3832 & 4.3482 & 0.0412 \\
\hline 4.0 & 12.3470 & -45.0 & 13.0455 & -49.5 & 64.5 & -4.0 & 4.8299 & 0.0403 \\
\hline \multicolumn{9}{|l|}{$\psi=270^{\circ}$} \\
\hline 0.1 & 0.9487 & -36.0 & 1.3130 & -77.0 & -91.0 & -23.1 & 1.2583 & 0.1641 \\
\hline 0.3 & 1.3074 & -36.0 & 1.9523 & -27.5 & -91.0 & -3.2 & 1.4404 & 0.1641 \\
\hline 0.5 & 1.7135 & -35.5 & 2.3435 & -16.5 & -91.0 & -6.1 & 1.6527 & 0.1641 \\
\hline 1.0 & 2.9251 & -35.0 & 3.3646 & -29.5 & -85.0 & -6.3 & 2.0921 & 0.0831 \\
\hline 1.5 & 4.4054 & -34.5 & 4.6268 & -32.5 & 53.0 & -14.9 & 2.6390 & 0.0325 \\
\hline 2.0 & 6.1469 & -34.5 & 6.4228 & -32.5 & 51.5 & -9.8 & 3.1206 & 0.0317 \\
\hline 2.5 & 8.1454 & -34.5 & 8.4714 & -32.5 & 50.0 & -6.1 & 3.6060 & 0.0311 \\
\hline 3.0 & 10.3987 & -34.5 & 10.7717 & -32.5 & 49.0 & -3.3 & 4.0926 & 0.0306 \\
\hline 3.5 & 12.9053 & -34.5 & 13.3232 & -32.5 & 48.0 & -1.1 & 4.5812 & 0.0302 \\
\hline 4.0 & 15.6644 & -34.5 & 16.1256 & -32.5 & 47.5 & 0.8 & 5.0689 & 0.0300 \\
\hline \multicolumn{9}{|l|}{$\psi=315^{\circ}$} \\
\hline 0.1 & 0.8041 & -11.0 & 0.8084 & -10.5 & 24.5 & -53.9 & 1.3212 & 0.0242 \\
\hline 0.3 & 1.3176 & -13.0 & 1.3323 & -12.0 & 27.0 & -46.9 & 1.5277 & 0.0246 \\
\hline 0.5 & 1.8650 & -14.0 & 1.8902 & -13.0 & 26.5 & -41.8 & 1.7330 & 0.0245 \\
\hline 1.0 & 3.3998 & -15.5 & 3.4483 & -14.0 & 27.0 & -32.8 & 2.2385 & 0.0246 \\
\hline 1.5 & 5.1807 & -16.5 & 5.2485 & -15.0 & 26.5 & -26.9 & 2.7388 & 0.0245 \\
\hline 2.0 & 7.2104 & -17.0 & 7.2948 & -15.0 & 26.0 & -23.1 & 3.2395 & 0.0244 \\
\hline 2.5 & 9.4896 & -17.0 & 9.5889 & -15.5 & 25.5 & -20.2 & 3.7363 & 0.0243 \\
\hline 3.0 & 12.0186 & -17.5 & 12.1315 & -15.5 & 25.0 & -18.1 & 4.2336 & 0.0242 \\
\hline 3.5 & 14.7973 & -17.5 & 14.9231 & -15.5 & 25.0 & -16.3 & 4.7286 & 0.0242 \\
\hline 4.0 & 17.8260 & -17.5 & 17.9640 & -16.0 & 24.5 & -14.6 & 5.2211 & 0.0242 \\
\hline
\end{tabular}

point. Comparing the maximum variations of energy for the case where the impulse is applied at the periapsis with the case where this location is a free parameter, it is possible to measure the benefits of the second type of maneuver. In general, for small values of the magnitude of the maneuver, which are the more practical cases, the differences are very large when optimizing or not the location of the impulse. Table 1 shows that, in most cases, the impulse dominates the maneuver and the location has smaller effects in the maneuver. The regions with more or less effects change with the geometry (angle of approach).

Those tables also show that the values of $\alpha$, in most of the cases, are negative. The reason is that, 

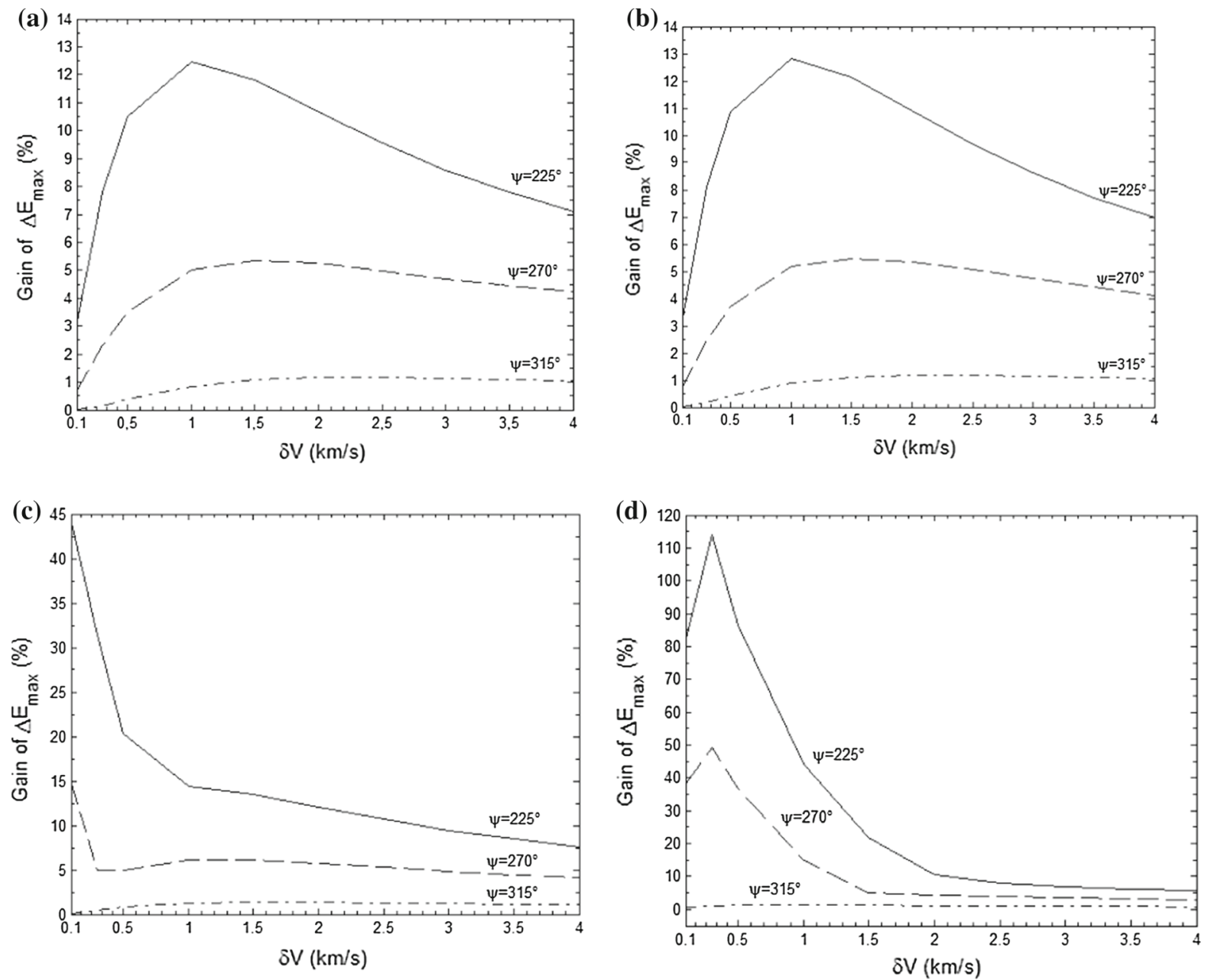

Fig. 8 Gain in the maximum energy variation for $\theta$ optimal compared to $\theta=0^{\circ} \mathbf{a} r_{p}=1.02 R_{m}, \mathbf{b} r_{p}=1.1 R_{m}, r_{p}=2.0 \mathrm{R}_{m}, \mathbf{d}$ $r_{p}=5.0 R_{m}$

for $-180^{\circ} \leq \alpha \leq 0^{\circ}$, there is a negative component from the impulse in the radial direction. This fact makes the spacecraft to get closer to the secondary body and, therefore, cause an increase in the energy variations.

It is also noted that, in several cases, $\theta$ is negative for the point of maximum variation of energy. It means that the impulse is applied before the spacecraft passage by the periapsis of the orbit, when the spacecraft is approaching $M_{2}$. This type of maneuver allows a better geometry for the interaction between the spacecraft and $M_{2}$, so making possible adjustments in the trajectory, to obtain the optimal point.

The percentage gain in the maximum energy variation for $\theta$ optimal compared with the case where the impulse is applied in the periapsis of the orbit $\left(\theta=0^{\circ}\right)$, for the same initial conditions and different periapsis distances, is shown next, in Fig. 8.

Note that, for all the situations studied, the gain is positive. It means that the impulse applied outside the periapsis of the orbit of the spacecraft around $M_{2}$ is the best option. The gain depends on the geometry of the maneuver.

The radius of the sphere of influence of the Moon is 0.172 c.u. [37]. The value $R$ is the distance between $M_{2}$ and the spacecraft when the impulse is applied. Comparing $R$ and the radius of influence of $M_{2}$, it is shown that the impulses were always applied inside the sphere of influence of $M_{2}$. 

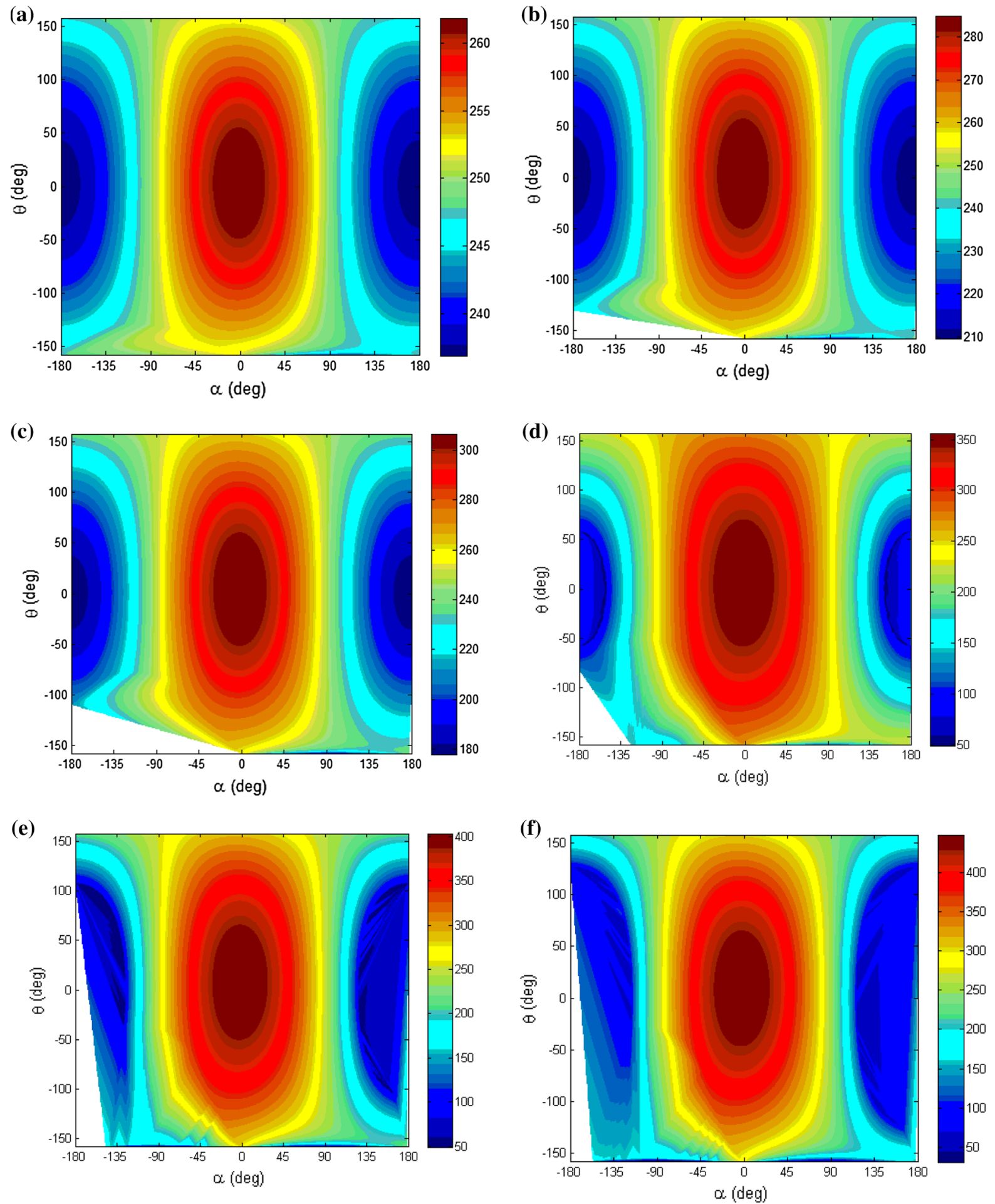

Fig. 9 Energy variation in $\mathrm{km}^{2} / \mathrm{s}^{2}$, for $r_{p}=1.02 R_{j}, \psi=270^{\circ}$ and different values for $\delta V$ a $\delta V=0.1 \mathrm{~km} / \mathrm{s}, \mathbf{b} \delta V=0.3 \mathrm{~km} / \mathrm{s}, \mathbf{c}$ $\delta V=0.5 \mathrm{~km} / \mathrm{s}, \mathbf{d} \delta V=1.0 \mathrm{~km} / \mathrm{s}, \mathbf{e} \delta V=1.5 \mathrm{~km} / \mathrm{s}, \mathbf{f} \delta V=2.0 \mathrm{~km} / \mathrm{s}$ 
(a)

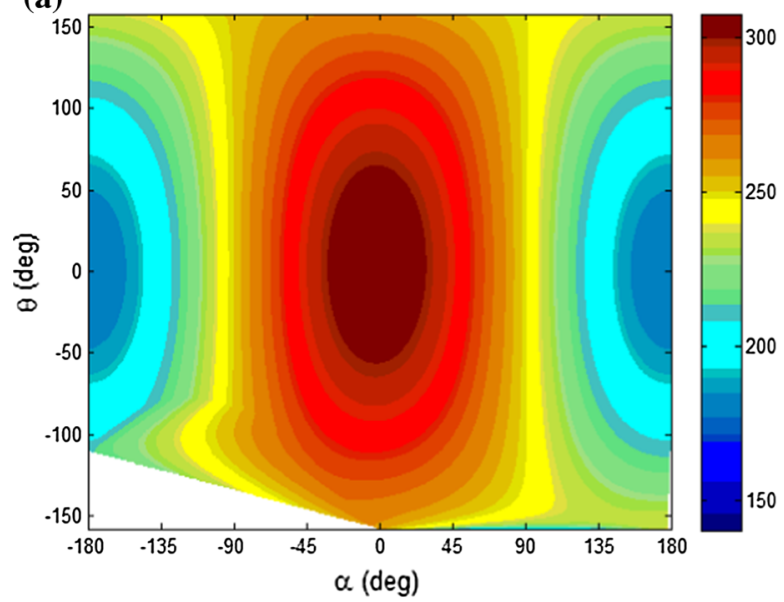

(b)

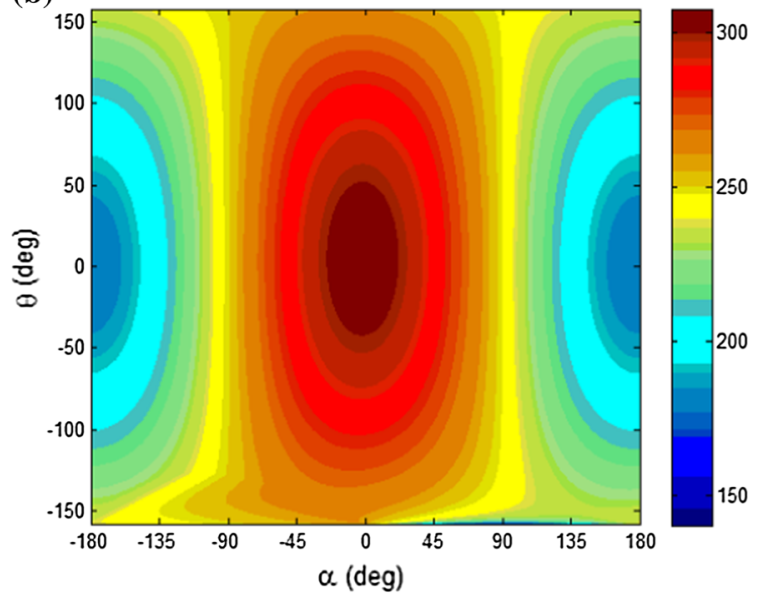

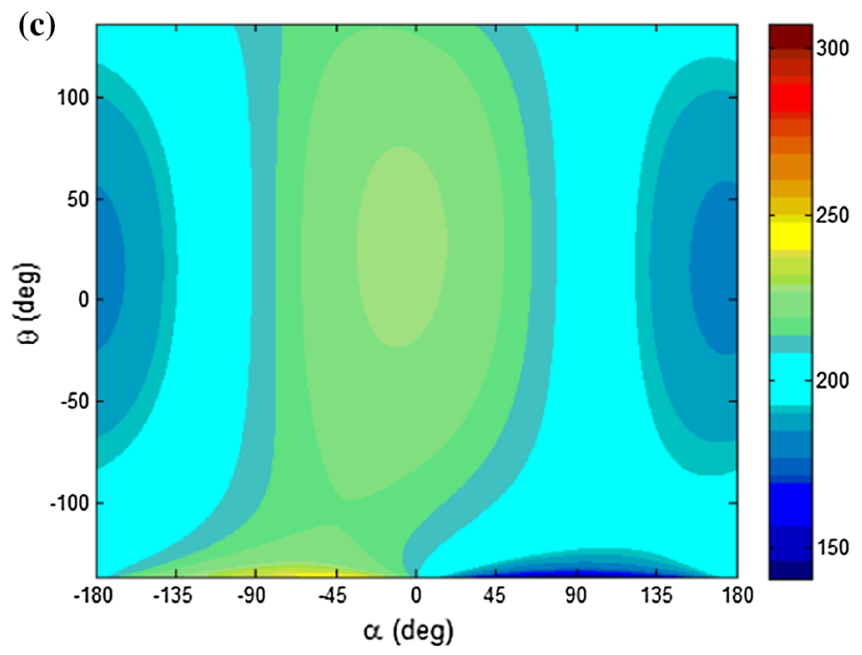

Fig. 10 Energy variation in $\mathrm{km}^{2} / \mathrm{s}^{2}$, for $\delta V=0.5 \mathrm{~km} / \mathrm{s}, \psi=270^{\circ}$ and different values of $r_{p}$ a $r_{p}=1.02 R_{j}, \mathbf{b} r_{p}=1.1 R_{j}$, c $r_{p}=5.0 R_{j}$

\subsection{Sun-Jupiter system}

Next, it is shown the results for the Sun-Jupiter system. The approach velocity $\left(V_{\infty_{-}}\right)$used in the simulations is 0.7633 c.u. Figure 9 shows the energy variation for $r_{p}=1.02 R_{j}, \psi=270^{\circ}$ and $\delta V=$ $0.1,0.3,0.5,1.0,1.5$ and $2.0 \mathrm{~km} / \mathrm{s}$. It is observed that the magnitude of the energy variation is significantly larger for this system, when compared to the EarthMoon system. As occurred in the previous case, the energy variation is directly proportional to the magnitude of the impulse, as expected. Compared to the previous case (Earth-Moon), it is also clear that the locations of the maximum variations of energy are now near the center of the plot $(0,0)$. This fact occurs because the impulse has smaller effects compared to the gravity part of the maneuver, since the mass of Jupiter is much larger than the mass of the Moon. But, when the magnitude of the impulse increases, this location goes to negative values of $\alpha$ and positive values of $\theta$. In other aspects, the behaviors are similar to the previous cases. The location of the impulse now has some effects in the borders, with the value zero given the more negative values for the variation of energy.

Figure 10 shows the variation of energy (on the same scale) for $\psi=270^{\circ}, \delta V=0.5 \mathrm{~km} / \mathrm{s}$ and different values for the periapsis radius $\left(r_{p}\right)$. It shows the decrease of the maximum energy variation when the spacecraft is passing at larger distances from the secondary body. 

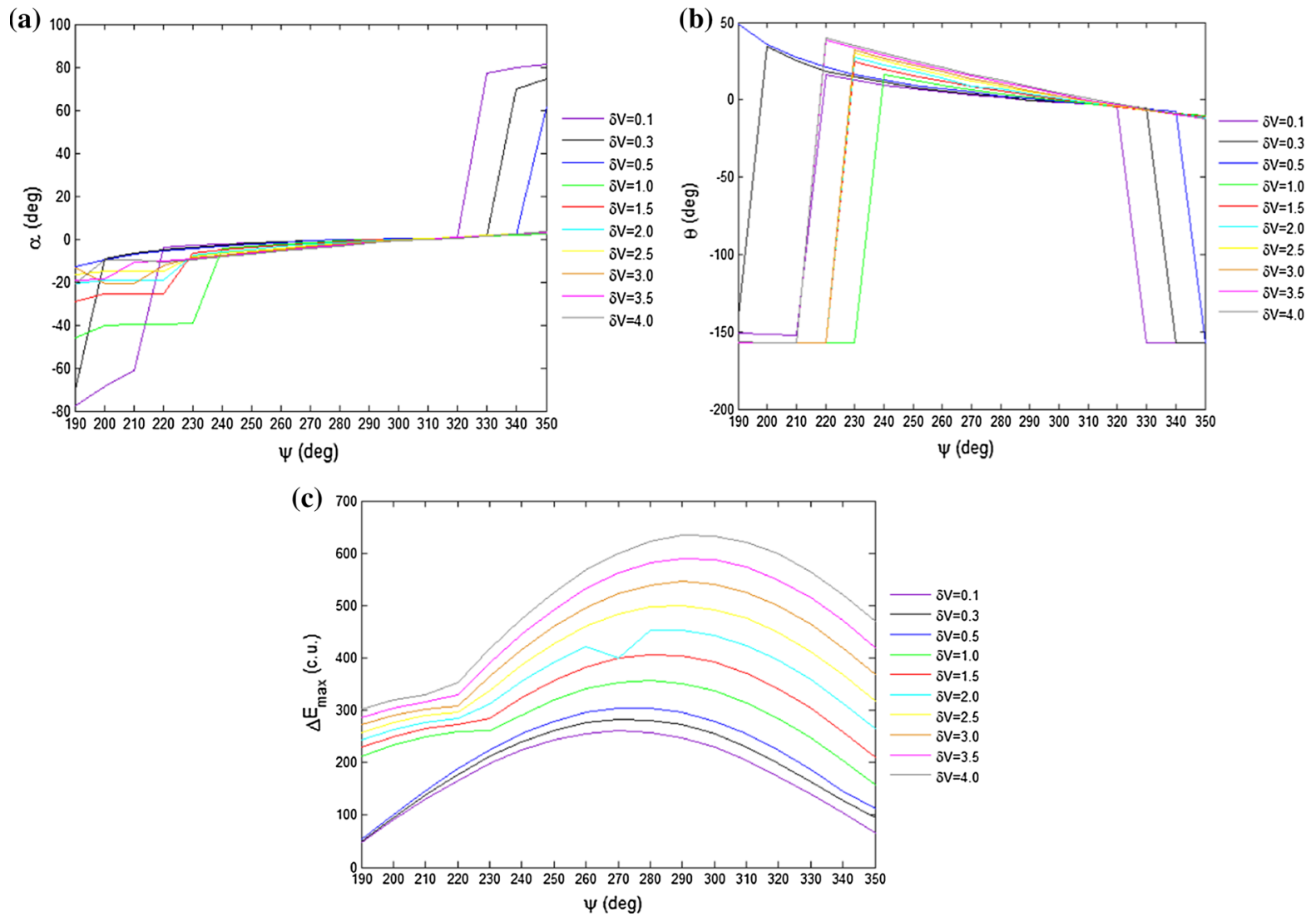

Fig. 11 a $\psi$ versus $\alpha$, b $\psi$ versus $\theta$ and $\mathbf{c} \psi$ versus $\Delta E_{\max }$ for $1.1 \mathrm{R}_{j}$

The blank regions in Figs. 9 and 10 also indicate collisions or captures of the spacecraft with respect to Jupiter. Note that, for periapsis distance equal to 1.1 and $5.0 R_{j}$, there is no capture or collision of the spacecraft.

The maximum variation of energy, for each condition, is shown in Figs. 11 and 12, for $r_{p}=1.1$ and 5.0 $R_{j}$, respectively. Figure (a) shows the angle of approach $(\psi)$ versus the angle that defines the direction of the impulse $(\alpha)$. Figure (b) shows the angle of approach $(\psi)$ versus the angle that defines the application point of the impulse $(\theta)$. Figure (c) shows the angle of approach $(\psi)$ versus the maximum variation of energy $\left(\Delta E_{\max }\right)$. All the cases used $\delta V=0.1$ to $\delta V=4.0 \mathrm{~km} / \mathrm{s}$. The results are similar to the previous cases, with the differences coming from the relative small effects of the impulse.

The empirical equations that describe the maximum energy variations for the Sun-Jupiter system, for $r_{p}=$
$1.1 R_{j}$ and the coefficients described in "Appendix 3", are as follows:

$$
\begin{aligned}
\Delta E_{\max }= & a_{3} \psi^{10}+b_{3} \psi^{9}+c_{3} \psi^{8}+d_{3} \psi^{7} \\
& +e_{3} \psi^{6}+f_{3} \psi^{5}+g_{3} \psi^{4}+h_{3} \psi^{3} \\
& +i_{3} \psi^{2}+j_{3} \psi+k_{3}
\end{aligned}
$$

Figure 12 shows the maximum energy variations and their respective data for $r_{p}=5.0 R_{j}$, and in the sequence, it is presented the equations that estimate this value. The coefficients of Eq. 23 are shown in "Appendix 3".

$$
\begin{aligned}
\Delta E_{\max }= & a_{4} \psi^{10}+b_{4} \psi^{9}+c_{4} \psi^{8}+d_{4} \psi^{7} \\
& +e_{4} \psi^{6}+f_{4} \psi^{5}+g_{4} \psi^{4}+h_{4} \psi^{3} \\
& +i_{4} \psi^{2}+j_{4} \psi+k_{4}
\end{aligned}
$$

Tables 5, 6 and 7, in "Appendix 2", show the maximum energy variations for $\theta=0$ and $\theta$ optimal, for 

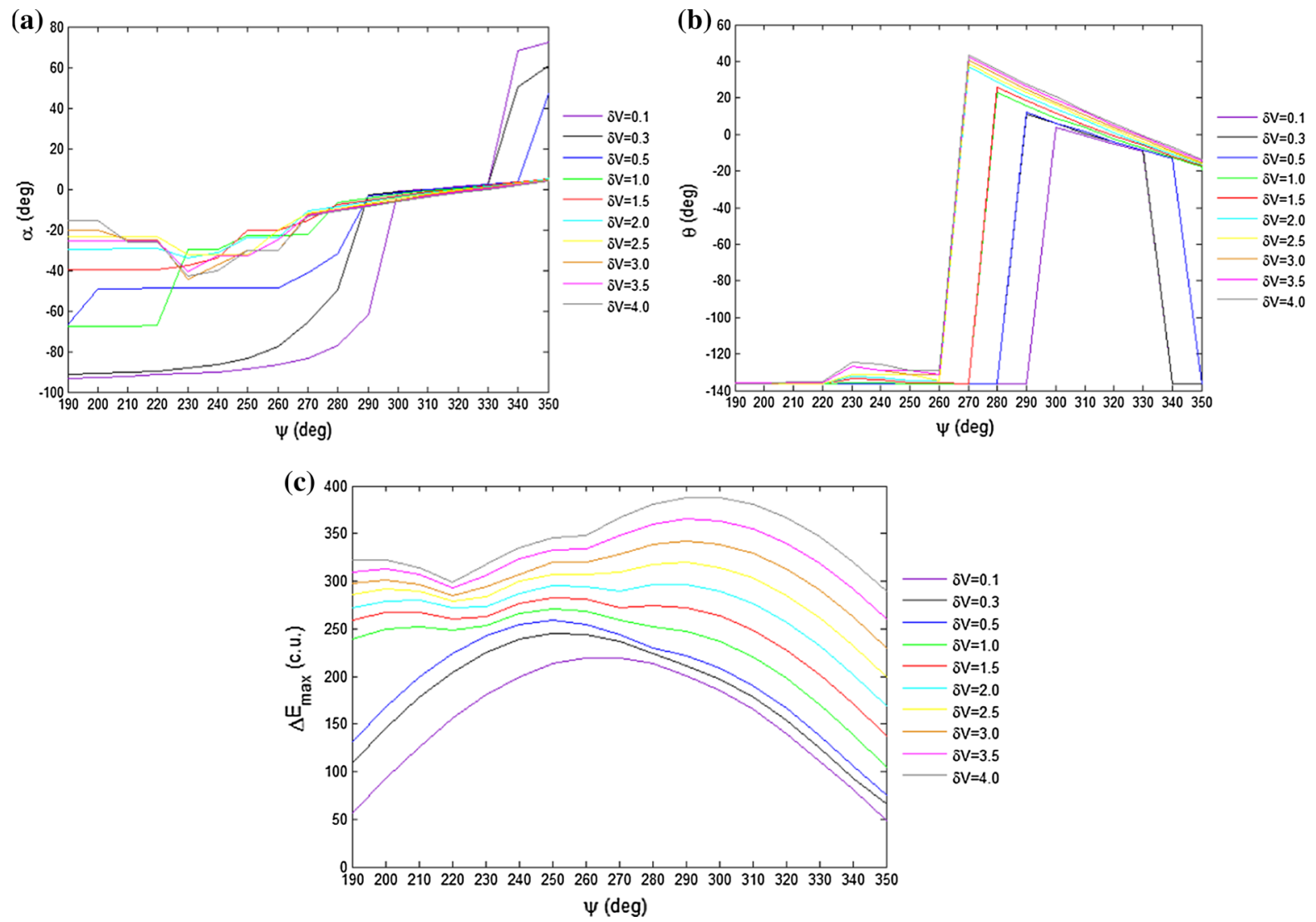

Fig. 12 a $\psi$ versus $\alpha, \mathbf{b} \psi$ versus $\theta$ and $\mathbf{c} \psi$ versus $\Delta E_{\max }$ for $5.0 R_{j}$

$r_{p}=1.02 R_{j}, 1.1 R_{j}$ and $5.0 R_{j}$, respectively. The same increases in the variation of energy obtained by optimizing the location of the impulse are noticed, with large magnitudes now. The same applications for those plots shown in the simulations for the Earth-Moon system are also valid here, with the horizontal and vertical lines given the same information described in that point.

The gain for the maximum energy variations considering the optimal point for the impulse application with respect to $\theta=0^{\circ}$ is shown in Fig. 13.

For $r_{p}=1.02 R_{j}$ and $r_{p}=1.1 R_{j}$, the gain for the angle of approach equal to $270^{\circ}$ and $315^{\circ}$ is from 0 to $1 \%$. For $r_{p}=5.0 R_{j}$ this interval is larger, from 0 to approximately $7.5 \%$. The best gains are for $\psi=225^{\circ}$ for each periapsis distance. The gains in maximum energy variations for optimal $\theta$ increase when the distance from the periapsis increases. The radius of the sphere of influence of Jupiter is 0.062 c.u. [37]. Note that, in all cases, the impulse was applied inside the sphere of influence of Jupiter.

\section{Conclusions}

In general, the results showed mappings that described the main effects of a powered Swing-By, in terms of the variation of energy as a function of the parameters involved in this maneuver. In particular, it shows the locations, in terms of the direction and the location of the impulse applied, for the solutions with maximum variations of energy. It is shown that they occur when the impulse is not applied in the tangential direction and in points which are not located in the periapsis of the orbits, as expected from the literature. The explanation for this fact is the effect of the gravity part of the maneuver combined with the impulse.

The organization of the results in plots showing the variations of energy, direction and location of the impulsive maneuver as a function of the angle of approach gives a new view of this maneuver and helps to plan a complete mission. Vertical lines give the minimum magnitude of the impulse required to get a given 
Table 5 Information about the maximum energy variation for $r_{p}=1.02 R_{j}$ in the Sun-Jupiter-spacecraft system

\begin{tabular}{|c|c|c|c|c|c|c|c|c|}
\hline$\delta V(\mathrm{~km} / \mathrm{s})$ & $\Delta E_{\max }\left(\mathrm{km}^{2} / \mathrm{s}^{2}\right)$, for $\theta=0^{\circ}$ & $\alpha\left(^{\circ}\right)$ for $\theta=0^{\circ}$ & $\Delta E_{\max }\left(\mathrm{km}^{2} / \mathrm{s}^{2}\right)$ & $\alpha\left(^{\circ}\right)$ & $\theta\left({ }^{\circ}\right)$ & $\zeta\left(^{\circ}\right)$ & $V_{\infty+}($ c.u. $)$ & $R$ (c.u.) \\
\hline \multicolumn{9}{|l|}{$\psi=225^{\circ}$} \\
\hline 0.1 & 183.4815 & -3.5 & 183.5259 & -3.5 & 13.1 & 9.5 & 1.1092 & 0.0001 \\
\hline 0.3 & 196.6664 & -4.0 & 196.8390 & -4.0 & 15.0 & 12.0 & 1.1709 & 0.0001 \\
\hline 0.5 & 209.0945 & -4.5 & 209.4492 & -4.5 & 17.5 & 13.8 & 1.2289 & 0.0001 \\
\hline 1.0 & 237.9061 & -6.0 & 255.2349 & -59.0 & -158.0 & 75.9 & 0.9120 & 0.0133 \\
\hline 1.5 & 264.6469 & -7.5 & 271.2379 & -35.5 & -158.0 & 84.5 & 0.9411 & 0.0133 \\
\hline 2.0 & 290.1508 & -8.5 & 293.4219 & -7.5 & 28.5 & 20.5 & 1.5901 & 0.0001 \\
\hline 2.5 & 314.8936 & -9.0 & 319.6123 & -8.0 & 30.6 & 21.4 & 1.6936 & 0.0001 \\
\hline 3.0 & 339.1763 & -10.0 & 345.5059 & -9.0 & 32.5 & 22.2 & 1.7893 & 0.0001 \\
\hline 3.5 & 363.1958 & -10.5 & 371.2656 & -9.5 & 34.0 & 22.7 & 1.8815 & 0.0001 \\
\hline 4.0 & 387.0919 & -11.0 & 397.0022 & -9.5 & 35.5 & 23.1 & 1.9699 & 0.0001 \\
\hline \multicolumn{9}{|l|}{$\psi=270^{\circ}$} \\
\hline 0.1 & 262.0352 & -0.5 & 262.0388 & -0.5 & 2.6 & -16.9 & 1.0651 & 0.0001 \\
\hline 0.3 & 285.4367 & -1.0 & 285.4527 & -1.0 & 3.5 & -12.7 & 1.1241 & 0.0001 \\
\hline 0.5 & 307.5417 & -1.0 & 307.5792 & -1.0 & 4.0 & -9.6 & 1.1818 & 0.0001 \\
\hline 1.0 & 358.7315 & -1.5 & 358.8749 & -1.5 & 6.1 & -4.4 & 1.3194 & 0.0001 \\
\hline 1.5 & 405.8705 & -2.0 & 406.2099 & -2.0 & 8.1 & -1.5 & 1.4475 & 0.0001 \\
\hline 2.0 & 450.2507 & -2.5 & 450.8897 & -2.5 & 9.6 & 0.5 & 1.5655 & 0.0001 \\
\hline 2.5 & 492.6379 & -3.0 & 493.6866 & -3.0 & 11.6 & 1.7 & 1.6769 & 0.0001 \\
\hline 3.0 & 533.5344 & -3.5 & 535.1019 & -3.5 & 13.0 & 2.5 & 1.7818 & 0.0001 \\
\hline 3.5 & 573.2904 & -4.0 & 575.4819 & -4.0 & 14.1 & 3.2 & 1.8808 & 0.0001 \\
\hline 4.0 & 612.1626 & -4.5 & 615.0798 & -4.0 & 15.5 & 3.6 & 1.9759 & 0.0001 \\
\hline \multicolumn{9}{|l|}{$\psi=315^{\circ}$} \\
\hline 0.1 & 190.4964 & 1.0 & 190.5014 & 1.0 & -3.1 & 10.2 & 0.8784 & 0.0001 \\
\hline 0.3 & 217.4980 & 1.0 & 217.5143 & 1.0 & -3.1 & 15.8 & 0.9482 & 0.0001 \\
\hline 0.5 & 243.4551 & 1.0 & 243.4832 & 1.0 & -3.5 & 19.7 & 1.0164 & 0.0001 \\
\hline 1.0 & 304.9972 & 1.0 & 305.0512 & 1.0 & -3.5 & 25.6 & 1.1749 & 0.0001 \\
\hline 1.5 & 363.0884 & 1.0 & 363.1581 & 1.0 & -3.1 & 28.7 & 1.3183 & 0.0001 \\
\hline 2.0 & 418.7093 & 1.0 & 418.7831 & 1.0 & -3.1 & 30.5 & 1.4497 & 0.0001 \\
\hline 2.5 & 472.4514 & 0.5 & 472.5188 & 0.5 & -2.6 & 31.4 & 1.5716 & 0.0001 \\
\hline 3.0 & 524.7111 & 0.5 & 524.7639 & 0.5 & -2.0 & 32.0 & 1.6857 & 0.0001 \\
\hline 3.5 & 575.7684 & 0.5 & 575.8037 & 0.5 & -1.6 & 32.3 & 1.7934 & 0.0001 \\
\hline 4.0 & 625.8366 & 0.5 & 625.8542 & 0.5 & -1.1 & 32.3 & 1.8959 & 0.0001 \\
\hline
\end{tabular}

energy variation and the maximum variation of energy for a given magnitude of the impulse. Horizontal lines indicate a fixed variation of energy, from where it is visible the value of the angle of approach that minimizes the magnitude of the impulse, which is not $270^{\circ}$, as one could expected. Those lines also give the minimum magnitude of the impulse for a given angle of approach. It helps to choose the most adequate parameters for a given mission. After that, the other plots give the point of application and the direction of the impulse.

The results showed here help to optimize the whole mission, by showing the energy variations as a function of the geometry of the maneuver. In that way, the effects of other constraints of the mission that impact in the incoming trajectories to $M_{2}$ can be evaluated and a balance between the goals of the mission can be done. 
Table 6 Information about the maximum energy variation for $r_{p}=1.1 R_{j}$ in the Sun-Jupiter-spacecraft system

\begin{tabular}{|c|c|c|c|c|c|c|c|c|}
\hline$\delta V(\mathrm{~km} / \mathrm{s})$ & $\begin{array}{l}\Delta E_{\max }\left(\mathrm{km}^{2} / \mathrm{s}^{2}\right), \\
\text { for } \theta=0^{\circ}\end{array}$ & $\alpha\left(^{\circ}\right)$ for $\theta=0^{\circ}$ & $\Delta E_{\max }\left(\mathrm{km}^{2} / \mathrm{s}^{2}\right)$ & $\alpha\left(^{\circ}\right)$ & $\theta\left(^{\circ}\right)$ & $\zeta\left(^{\circ}\right)$ & $V_{\infty+}($ c.u. $)$ & $R$ (c.u.) \\
\hline \multicolumn{9}{|l|}{$\psi=225^{\circ}$} \\
\hline 0.1 & 182.3517 & -4.0 & 182.4022 & -3.5 & 14.0 & 7.6 & 1.1079 & 0.0001 \\
\hline 0.3 & 194.7724 & -4.5 & 194.9666 & -4.5 & 16.5 & 10.0 & 1.1671 & 0.0001 \\
\hline 0.5 & 206.4901 & -5.0 & 206.8863 & -5.0 & 18.5 & 11.8 & 1.2225 & 0.0001 \\
\hline 1.0 & 233.6808 & -6.5 & 261.6039 & -39.0 & -157.5 & 76.8 & 0.9236 & 0.0183 \\
\hline 1.5 & 258.9480 & -8.0 & 274.3658 & -25.0 & -157.5 & 83.8 & 0.9471 & 0.0183 \\
\hline 2.0 & 283.0748 & -9.0 & 286.6224 & -8.0 & 30.0 & 18.3 & 1.5728 & 0.0001 \\
\hline 2.5 & 306.5114 & -10.0 & 311.5987 & -9.0 & 32.6 & 19.3 & 1.6715 & 0.0001 \\
\hline 3.0 & 329.5400 & -10.5 & 336.3335 & -9.5 & 34.5 & 20.1 & 1.7655 & 0.0001 \\
\hline 3.5 & 352.3511 & -11.5 & 360.9756 & -10.0 & 36.0 & 20.6 & 1.8550 & 0.0001 \\
\hline 4.0 & 375.0731 & -12.0 & 385.6259 & -10.5 & 37.6 & 21.2 & 1.9394 & 0.0001 \\
\hline \multicolumn{9}{|l|}{$\psi=270^{\circ}$} \\
\hline 0.1 & 260.5056 & -1.0 & 260.5096 & -1.0 & 3.1 & -17.9 & 1.0665 & 0.0001 \\
\hline 0.3 & 282.9395 & -1.0 & 282.9584 & -1.0 & 3.6 & -13.8 & 1.1229 & 0.0001 \\
\hline 0.5 & 304.1496 & -1.0 & 304.1930 & -1.0 & 5.0 & -10.8 & 1.1787 & 0.0001 \\
\hline 1.0 & 353.3039 & -2.0 & 353.4658 & -2.0 & 6.6 & -5.8 & 1.3123 & 0.0001 \\
\hline 1.5 & 398.5886 & -2.5 & 398.9678 & -2.5 & 8.5 & -2.9 & 1.4362 & 0.0001 \\
\hline 2.0 & 441.2305 & -3.0 & 441.9393 & -3.0 & 10.5 & -1.0 & 1.5512 & 0.0001 \\
\hline 2.5 & 481.9640 & -3.5 & 483.1186 & -3.0 & 12.1 & 0.2 & 1.6597 & 0.0001 \\
\hline 3.0 & 521.2716 & -4.0 & 522.9899 & -3.5 & 13.6 & 1.2 & 1.7611 & 0.0001 \\
\hline 3.5 & 559.4924 & -4.5 & 561.8853 & -4.0 & 15.5 & 1.8 & 1.857 & 0.0001 \\
\hline 4.0 & 596.8740 & -5.0 & 600.0466 & -4.5 & 16.5 & 2.3 & 1.950. & 0.0001 \\
\hline \multicolumn{9}{|l|}{$\psi=315^{\circ}$} \\
\hline 0.1 & 189.3229 & 1.0 & 189.3285 & 1.0 & -3.5 & 8.9 & 0.8806 & 0.0001 \\
\hline 0.3 & 215.4730 & 1.0 & 215.4898 & 1.0 & -3.5 & 14.3 & 0.9479 & 0.0001 \\
\hline 0.5 & 240.6286 & 1.0 & 240.6571 & 1.0 & -3.5 & 18.2 & 1.0135 & 0.0001 \\
\hline 1.0 & 300.2986 & 1.0 & 300.3523 & 1.0 & -3.5 & 24.0 & 1.1671 & 0.0001 \\
\hline 1.5 & 356.6313 & 1.0 & 356.6991 & 1.0 & -3.5 & 27.1 & 1.3068 & 0.0001 \\
\hline 2.0 & 410.5637 & 1.0 & 410.6329 & 1.0 & -3.1 & 28.8 & 1.4350 & 0.0001 \\
\hline 2.5 & 462.6667 & 0.5 & 462.7274 & 0.5 & -2.6 & 29.8 & 1.5539 & 0.0001 \\
\hline 3.0 & 513.3234 & 0.5 & 513.3687 & 0.5 & -2.1 & 30.4 & 1.6654 & 0.0001 \\
\hline 3.5 & 562.8089 & 0.5 & 562.8355 & 0.5 & -1.5 & 30.7 & 1.7709 & 0.0001 \\
\hline 4.0 & 611.3303 & 0.0 & 611.3412 & 0.0 & -1.0 & 30.8 & 1.8711 & 0.0001 \\
\hline
\end{tabular}

Besides that, the results showed here also help to correct errors in the arriving trajectory. They can find the maximum variation of energy, the magnitude, direction and location of the impulse, as a function of the new actual angle of approach.

The results detailed and confirmed that the optimum maneuver is not at the periapsis and aligned to the velocity of the spacecraft. The reason is the combined effect of the impulsive maneuver with the Swing-By. Applying the impulse in different positions and in different directions changes the main parameters of the Swing-By. The periapsis distance will be different, as well as the deflection angle. In this way, the loss of energy coming from the fact that the impulsive maneuver is not applied in the geometry that gets the most energy possible is compensated by the extra gain due 
Table 7 Information about the maximum energy variation for $r_{p}=5.0 R_{j}$ in the Sun-Jupiter-spacecraft system

\begin{tabular}{|c|c|c|c|c|c|c|c|c|}
\hline$\delta V(\mathrm{~km} / \mathrm{s})$ & $\Delta E_{\max }\left(\mathrm{km}^{2} / \mathrm{s}^{2}\right)$, for $\theta=0^{\circ}$ & $\alpha\left(^{\circ}\right)$ for $\theta=0^{\circ}$ & $\Delta E_{\max }\left(\mathrm{km}^{2} / \mathrm{s}^{2}\right)$ & $\alpha\left(^{\circ}\right)$ & $\theta\left(^{\circ}\right)$ & $\zeta\left(^{\circ}\right)$ & $V_{\infty+}($ c.u. $)$ & $R$ (c.u.) \\
\hline \multicolumn{9}{|l|}{$\psi=225^{\circ}$} \\
\hline 0.1 & 147.8296 & -35.5 & 168.7364 & -91.5 & -136.5 & -35.3 & 1.0658 & 0.0206 \\
\hline 0.3 & 150.6772 & -37.5 & 215.5769 & -89.0 & -136.5 & -18.0 & 1.0816 & 0.0206 \\
\hline 0.5 & 153.5374 & -38.5 & 234.3809 & -48.5 & -136.5 & -11.2 & 1.0961 & 0.0206 \\
\hline 1.0 & 160.8007 & -40.5 & 244.0048 & -35.0 & -135.5 & -9.3 & 1.1260 & 0.0144 \\
\hline 1.5 & 168.2862 & -41.5 & 253.6129 & -39.5 & -136.5 & 78.2 & 0.8645 & 0.0206 \\
\hline 2.0 & 176.0348 & -42.5 & 264.3384 & -29.0 & -136.5 & 84.3 & 0.9107 & 0.0206 \\
\hline 2.5 & 184.0695 & -42.5 & 273.1743 & -43.0 & -129.0 & -3.8 & 1.2051 & 0.0056 \\
\hline 3.0 & 192.4022 & -43.0 & 283.7682 & -44.5 & -127.0 & -2.1 & 1.2336 & 0.0047 \\
\hline 3.5 & 201.0424 & -43.0 & 294.4068 & -41.0 & -126.5 & -1.3 & 1.2733 & 0.0045 \\
\hline 4.0 & 209.9940 & -43.0 & 305.2776 & -43.0 & -124.5 & 0.1 & 1.3033 & 0.0039 \\
\hline \multicolumn{9}{|l|}{$\psi=270^{\circ}$} \\
\hline 0.1 & 211.7407 & -7.0 & 219.8661 & -83.5 & -136.5 & -33.0 & 1.0592 & 0.0207 \\
\hline 0.3 & 220.3136 & -8.0 & 236.6536 & -65.5 & -136.5 & -18.6 & 1.0153 & 0.0207 \\
\hline 0.5 & 228.5964 & -8.5 & 244.3214 & -41.5 & -136.5 & -12.7 & 1.0075 & 0.0207 \\
\hline 1.0 & 248.3450 & -10.5 & 258.4967 & -22.5 & -136.5 & -6.7 & 1.0244 & 0.0207 \\
\hline 1.5 & 267.1440 & -12.0 & 271.7682 & -15.5 & -136.5 & -4.9 & 1.0583 & 0.0207 \\
\hline 2.0 & 285.3485 & -13.5 & 289.4412 & -11.0 & 37.0 & -28.8 & 1.3344 & 0.0005 \\
\hline 2.5 & 303.1905 & -15.0 & 308.8418 & -12.0 & 39.0 & -27.1 & 1.3930 & 0.0005 \\
\hline 3.0 & 320.8294 & -16.0 & 328.1579 & -12.5 & 40.5 & -25.6 & 1.4499 & 0.0005 \\
\hline 3.5 & 338.3743 & -17.0 & 347.4695 & -13.0 & 42.0 & -24.2 & 1.5049 & 0.0005 \\
\hline 4.0 & 355.9066 & -17.5 & 366.8341 & -13.5 & 43.5 & -22.9 & 1.5575 & 0.0005 \\
\hline \multicolumn{9}{|l|}{$\psi=315^{\circ}$} \\
\hline 0.1 & 153.1102 & 0.5 & 153.1120 & 0.5 & -2.5 & -22.8 & 0.9612 & 0.0005 \\
\hline 0.3 & 166.2673 & 0.5 & 166.2702 & -0.5 & -2.0 & -20.4 & 0.9932 & 0.0005 \\
\hline 0.5 & 179.0641 & 0.5 & 179.0659 & 0.5 & -1.5 & -18.4 & 1.0253 & 0.0005 \\
\hline 1.0 & 209.7650 & 0.0 & 209.7653 & 0.0 & 0.5 & -14.5 & 1.1033 & 0.0005 \\
\hline 1.5 & 239.0249 & -0.5 & 239.0417 & -0.5 & 2.5 & -11.7 & 1.1785 & 0.0005 \\
\hline 2.0 & 267.2117 & -1.0 & 267.2795 & -1.0 & 4.0 & -9.7 & 1.2513 & 0.0005 \\
\hline 2.5 & 294.5898 & -2.0 & 294.7520 & -1.5 & 5.6 & -8.0 & 1.3211 & 0.0005 \\
\hline 3.0 & 321.3548 & -2.5 & 321.6600 & -2.0 & 7.0 & -6.6 & 1.3881 & 0.0005 \\
\hline 3.5 & 347.6561 & -3.0 & 348.1538 & -2.5 & 8.5 & -5.5 & 1.4530 & 0.0005 \\
\hline 4.0 & 373.6112 & -3.5 & 374.3482 & -3.0 & 9.5 & -4.4 & 1.5155 & 0.0005 \\
\hline
\end{tabular}

to the Swing-By to be performed with a large value of the deflection angle and a smaller value for the periapsis distance.

Some of the results shown here are expected from the "patched-conics" model, but accurate measurements need the better numerical model developed here. In particular, the results for the Earth-Moon system are subjected to large errors, due to the high mass parameter of the system. Some special and important cases, like captures, collisions and maneuvers where the spacecraft goes to larger distances from $M_{2}$, are not correctly identified by the "patched-conics" model, because the spacecraft spends too much time in intermediate regions that are not highly dominated by one of the primaries. It means that the variations of energy for each initial conditions will not be corrected, and even the locations of the points of maximum and minimum variations of energy will not be corrected. 

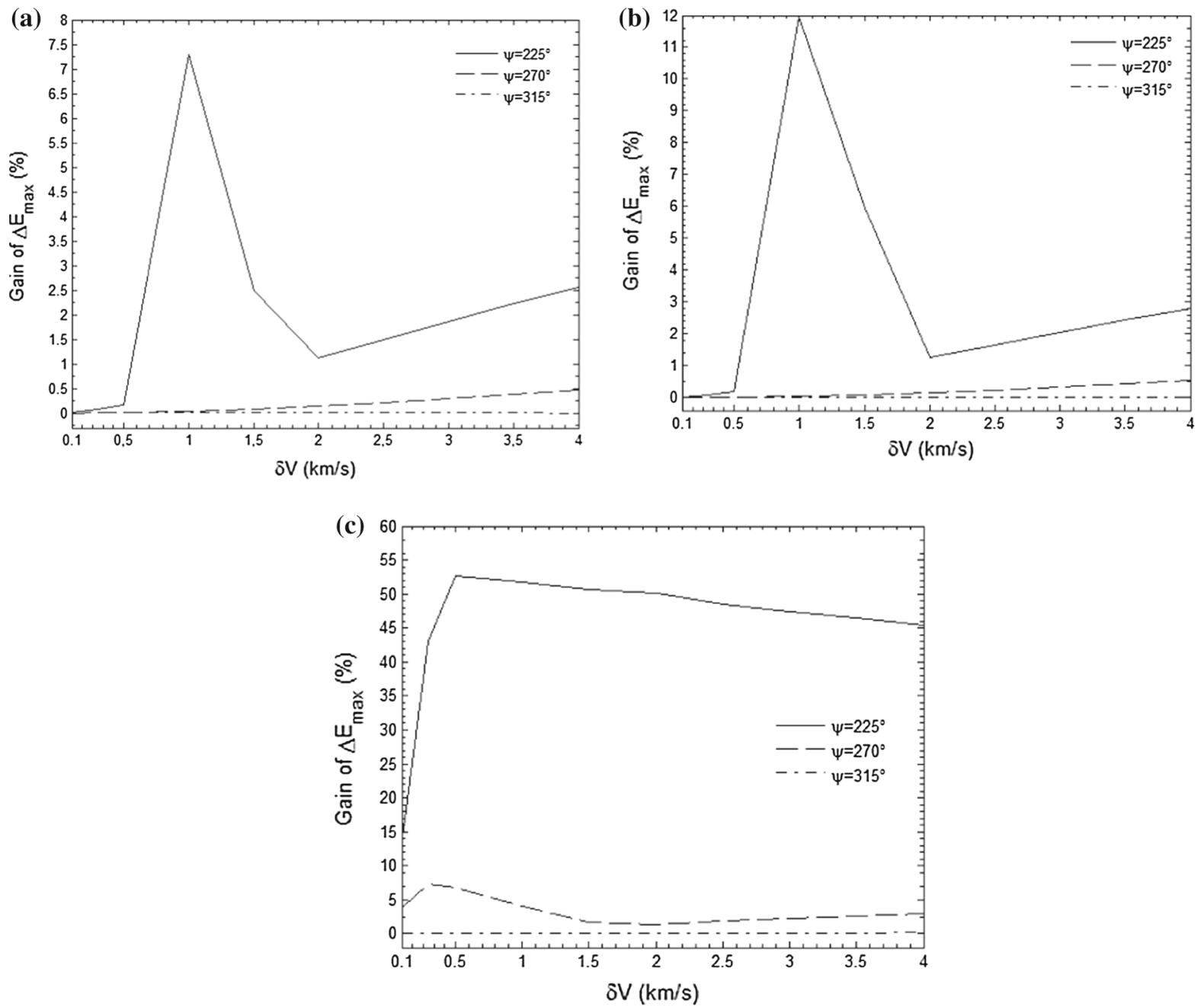

Fig. 13 Gain in the maximum energy variation for $\theta$ optimal when compared to $\theta=0 \mathbf{a} r_{p}=1.02 R_{j}, \mathbf{b} r_{p}=1.1 R_{j}$, $\mathbf{c} r_{p}=5.0 R_{j}$

Acknowledgements The authors wish to express their appreciation for the support provided by Grants \# 304700/2009-6 from the National Council for Scientific and Technological Development (CNPq); Grants \# 2011/08171-3 and 2014/06688-7, from São Paulo Research Foundation (FAPESP) and the financial support from the National Council for the Improvement of Higher Education (CAPES).

\section{Appendix 1: Detailed information about the trajec- tories in the Earth-Moon system}

It is shown here the maximum energy variations $\left(\Delta E_{\max }\right)$ and its corresponding data, as the true anomaly of the point where the impulse is applied $(\theta)$, the angle that gives the direction of the impulse $(\alpha)$, the deflection angle $(\zeta)$, the escape velocity $\left(V_{\infty+}\right)$ and $R$, which is the distance between the spacecraft and the secondary body at the instant that the impulse is applied.

\section{Appendix 2: Details of the trajectories for the Sun- Jupiter system}

It is shown here the maximum energy variations $\left(\Delta E_{\max }\right)$ and its corresponding data, as the true anomaly of the point where the impulse is applied $(\theta)$, the angle that gives the direction of the impulse $(\alpha)$, the deflection angle $(\zeta)$, the escape velocity $\left(V_{\infty}+\right)$ and $R$, which is the distance between the spacecraft 
and the secondary body at the instant that the impulse is applied.

\section{Appendix 3: Coefficients of the empirical equations that describe the maximum energy variation}

Equations (24) and (28) describe the coefficients of Eq. 20 as a function of the magnitude of the impulse $(\delta V)$.

$$
\begin{aligned}
a_{1}= & -\left(6.9093 \times 10^{-10}\right) \delta V^{8}+\left(1.2491 \times 10^{-8}\right) \delta V^{7} \\
& -\left(9.4632 \times 10^{-8}\right) \delta V^{6}+\left(3.8896 \times 10^{-7}\right) \delta V^{5} \\
& -\left(9.3631 \times 10^{-7}\right) \delta V^{4}+\left(1.3226 \times 10^{-6}\right) \delta V^{3} \\
& -\left(1.0262 \times 10^{-6}\right) \delta V^{2}+\left(3.6004 \times 10^{-7}\right) \delta V \\
& -\left(2.1218 \times 10^{-8}\right) \\
b_{1}= & \left(8.1943 \times 10^{-7}\right) \delta V^{8}-\left(1.4749 \times 10^{-5}\right) \delta V^{7} \\
& +0.00011121 \delta V^{6}-0.00045486 \delta V^{5} \\
& +0.0010895 \delta V^{4}-0.0015315 \delta V^{3} \\
& +0.0011831 \delta V^{2}-0.00041416 \delta V \\
& +\left(2.4623 \times 10^{-5}\right) \\
c_{1}= & -0.00036168 \delta V^{8}+0.0064807 \delta V^{7} \\
& -0.048632 \delta V^{6}+0.19791 \delta V^{5}-0.47159 \delta V^{4} \\
& +0.65953 \delta V^{3}-0.50708 \delta V^{2}+0.17702 \delta V \\
& -0.010831 \\
d_{1}= & 0.07033 \delta V^{8}-1.2546 \delta V^{7}+9.3694 \delta V^{6} \\
& -37.935 \delta V^{5}+89.917 \delta V^{4}-125.08 \delta V^{3} \\
& +95.691 \delta V^{2}-33.267 \delta V+2.1306 \\
e_{1}= & -0.74231 \delta V^{9}+7.6992 \delta V^{8}-1.701 \delta V^{7} \\
& -311.82 \delta V^{6}+1875.7 \delta V^{5}-5229 \delta V^{4} \\
& +7892.5 \delta V^{3}-6304.6 \delta V^{2}+2239.9 \delta V-151.11
\end{aligned}
$$

Equations (29) and (33) describe the coefficients of Eq. (21) as a function of the magnitude of the impulse $(\delta V)$.

$$
\begin{aligned}
a_{2}= & -\left(2.0996 \times 10^{-10}\right) \delta V^{8}+\left(3.7802 \times 10^{-9}\right) \delta V^{7} \\
& -\left(2.8519 \times 10^{-8}\right) \delta V^{6}+\left(1.1676 \times 10^{-7}\right) \delta V^{5} \\
& -\left(2.8016 \times 10^{-7}\right) \delta V^{4}+\left(3.951 \times 10^{-7}\right) \delta V^{3} \\
& -\left(3.071 \times 10^{-7}\right) \delta V^{2}+\left(1.0911 \times 10^{-7}\right) \delta V \\
& -\left(5.1707 \times 10^{-9}\right) \\
b_{2}= & \left(2.2047 \times 10^{-7}\right) \delta V^{8}-\left(4.0258 \times 10^{-6}\right) \delta V^{7}
\end{aligned}
$$

$$
\begin{aligned}
& +\left(3.0831 \times 10^{-5}\right) \delta V^{6}-0.00012824 \delta V^{5} \\
& +0.00031282 \delta V^{4}-0.00044882 \delta V^{3} \\
& +0.00035537 \delta V^{2}-0.00012942 \delta V \\
& +\left(7.0061 \times 10^{-6}\right) \\
c_{2}= & -\left(7.2312 \times 10^{-5}\right) \delta V^{9}+0.0011587 \delta V^{8} \\
& -0.0073561 \delta V^{7}+0.022503 \delta V^{6}-0.027767 \delta V^{5} \\
& -0.019652 \delta V^{4}+0.10125 \delta V^{3} \\
& -0.11473 \delta V^{2}+0.049405 \delta V-0.0031216 \\
d_{2}= & 0.016556 \delta V^{9}-0.26995 \delta V^{8}+1.7661 \delta V^{7} \\
& -5.7542 \delta V^{6}+8.7551-1.0826 \delta V^{4}-15.562 \delta V^{3} \\
& +20.553 \delta V^{2}-9.344 \delta V+0.70129 \\
e_{2}= & -1.3364 \delta V^{9}+22.003 \delta V^{8}-146.32 \delta V^{7} \\
& +492.14 \delta V^{6}-817.07 \delta V^{5}+345.12 \delta V^{4} \\
& +902.47 \delta V^{3}-1390.6 \delta V^{2}+659.13 \delta V-56.513
\end{aligned}
$$

Equations (34) and (44) describe the coefficients of Eq. (22) as a function of the magnitude of the impulse $(\delta V)$.

$$
\begin{aligned}
a_{3}= & \left(1.6042 \times 10^{-17}\right) \delta V^{8}-\left(2.5231 \times 10^{-16}\right) \delta V^{7} \\
& +\left(1.6116 \times 10^{-15}\right) \delta V^{6}-\left(5.3638 \times 10^{-15}\right) \delta V^{5} \\
& +\left(9.9236 \times 10^{-15}\right) \delta V^{4}-\left(1.0104 \times 10^{-14}\right) \delta V^{3} \\
& +\left(5.3072 \times 10^{-15}\right) \delta V^{2}-\left(1.2438 \times 10^{-15}\right) \delta V \\
& +\left(8.7211 \times 10^{-17}\right) \\
b_{3}= & -\left(4.3206 \times 10^{-14}\right) \delta V^{8}+\left(6.7949 \times 10^{-13}\right) \delta V^{7} \\
& -\left(4.3399 \times 10^{-12}\right) \delta V^{6}+\left(1.4442 \times 10^{-11}\right) \delta V^{5} \\
& -\left(2.6716 \times 10^{-11}\right) \delta V^{4}+\left(2.72 \times 10^{-11}\right) \delta V^{3} \\
& -\left(1.4287 \times 10^{-11}\right) \delta V^{2}+\left(3.3473 \times 10^{-12}\right) \delta V \\
& -\left(2.3467 \times 10^{-13}\right) \\
c_{3}= & \left(5.2025 \times 10^{-11}\right) \delta V^{8}-\left(8.1812 \times 10^{-10}\right) \delta V^{7} \\
& +\left(5.2248 \times 10^{-9}\right) \delta V^{6}-\left(1.7385 \times 10^{-8}\right) \delta V^{5} \\
& +\left(3.2158 \times 10^{-8}\right) \delta V^{4}-\left(3.2737 \times 10^{-8}\right) \delta V^{3} \\
& +\left(1.7197 \times 10^{-8}\right) \delta V^{2}-\left(4.0281 \times 10^{-9}\right) \delta V \\
& +\left(2.8236 \times 10^{-10}\right) \\
d_{3}= & -\left(3.6871 \times 10^{-8}\right) \delta V^{8}+\left(5.7978 \times 10^{-7}\right) \delta V^{7}
\end{aligned}
$$


$-\left(3.7024 \times 10^{-6}\right) \delta V^{6}+\left(1.2318 \times 10^{-5}\right) \delta V^{5}$

$-\left(2.2783 \times 10^{-5}\right) \delta V^{4}+\left(2.3193 \times 10^{-5}\right) \delta V^{3}$

$-\left(1.2185 \times 10^{-5}\right) \delta V^{2}+\left(2.8537 \times 10^{-6}\right) \delta V$

$-\left(2.0002 \times 10^{-7}\right)$

$e_{3}=\left(1.7033 \times 10^{-5}\right) \delta V^{8}-0.00026781 \delta V^{7}$

$+0.00171 \delta V^{6}-0.005689 \delta V^{5}+0.010521 \delta V^{4}$

$-0.01071 \delta V^{3}+0.0056279 \delta V^{2}$

$-0.001318 \delta V+\left(9.2376 \times 10^{-5}\right)$

$f_{3}=-0.0066105 \delta V^{9}+0.10848 \delta V^{8}$

$-0.7349 \delta V^{7}+2.6606 \delta V^{6}$

$-5.5748 \delta V^{5}+6.8616 \delta V^{4}$

$-4.8323 \delta V^{3}+1.8036 \delta V^{2}$

$-0.29959 \delta V+0.014695$

$g_{3}=1.4437 \delta V^{9}-23.699 \delta V^{8}$

$+160.63 \delta V^{7}-581.9 \delta V^{6}+1220.3 \delta V^{5}$

$-1503.8 \delta V^{4}+1060.6 \delta V^{3}$

$-396.53 \delta V^{2}+66.039 \delta V-3.2517$

$h_{3}=-214.76 \delta V^{9}+3526.6 \delta V^{8}-23914 \delta V^{7}$

$+86691 \delta V^{6}-\left(1.8196 \times 10^{-5}\right) \delta V^{5}$

$+\left(2.245 \times 10^{-5}\right) \delta V^{4}-\left(1.5858 \times 10^{-5}\right) \delta V^{3}$

$+59393 \delta V^{2}-9916.8 \delta+490.14$

$i_{3}=20824 \delta V^{9}-\left(3.4209 \times 10^{-5}\right) \delta V^{8}$

$+\left(2.3209 \times 10^{-6}\right) \delta V^{7}-\left(8.4193 \times 10^{-6}\right) \delta V^{6}$

$+\left(1.7688 \times 10^{-7}\right) \delta V^{5}-\left(2.1851 \times 10^{-7}\right) \delta V^{4}$

$+\left(1.5459 \times 10^{-7}\right) \delta V^{3}-\left(5.8 \times 10^{-6}\right) \delta V^{2}$

$+\left(9.7087 \times 10^{-5}\right) \delta V-48163$

$$
j_{3}=-\left(1.1884 \times 10^{-6}\right) \delta V^{9}+\left(1.953 \times 10^{-7}\right) \delta V^{8}
$$

$-\left(1.3257 \times 10^{-8}\right) \delta V^{7}+\left(4.8126 \times 10^{-8}\right) \delta V^{6}$

$-\left(1.0121 \times 10^{-9}\right) \delta V^{5}+\left(1.2518 \times 10^{-9}\right) \delta V^{4}$

$-\left(8.8709 \times 10^{-8}\right) \delta V^{3}+\left(3.334 \times 10^{-8}\right) \delta V^{2}$

$-\left(5.5947 \times 10^{-7}\right) \delta V+\left(2.7855 \times 10^{-6}\right)$

$k_{3}=\left(3.0312 \times 10^{-7}\right) \delta V^{9}-\left(4.9835 \times 10^{-8}\right) \delta V^{8}$

$+\left(3.3847 \times 10^{-9}\right) \delta V^{7}-\left(1.2296 \times 10^{-10}\right) \delta V^{6}$

$+\left(2.5884 \times 10^{-10}\right) \delta V^{5}-\left(3.2059 \times 10^{-10}\right) \delta V^{4}$

$$
\begin{aligned}
& +\left(2.2755 \times 10^{-10}\right) \delta V^{3}-\left(8.5671 \times 10^{-9}\right) \delta V^{2} \\
& +\left(1.4412 \times 10^{-9}\right) \delta V-\left(7.2007 \times 10^{-7}\right)
\end{aligned}
$$

Equations (45) and (55) describe the coefficients of Eq. (23) as a function of the magnitude of the impulse $(\delta V)$.

$$
\begin{aligned}
a_{4}= & -\left(1.0705 \times 10^{-18}\right) \delta V^{9}+\left(1.6883 \times 10^{-17}\right) \delta V^{8} \\
& -\left(1.1045 \times 10^{-16}\right) \delta V^{7}+\left(3.9381 \times 10^{-16}\right) \delta V^{6} \\
& -\left(8.5765 \times 10^{-16}\right) \delta V^{5}+\left(1.2342 \times 10^{-15}\right) \delta V^{4} \\
& -\left(1.229 \times 10^{-15}\right) \delta V^{3}+\left(7.7792 \times 10^{-16}\right) \delta V^{2} \\
& -\left(1.9375 \times 10^{-16}\right) \delta V+\left(9.8868 \times 10^{-18}\right)
\end{aligned}
$$

$b_{4}=\left(4.2985 \times 10^{-15}\right) \delta V^{8}-\left(6.1705 \times 10^{-14}\right) \delta V^{7}$

$+\left(3.4654 \times 10^{-13}\right) \delta V^{6}-\left(9.4239 \times 10^{-13}\right) \delta V^{5}$

$+\left(1.1863 \times 10^{-12}\right) \delta V^{4}-\left(3.4127 \times 10^{-13}\right) \delta V^{3}$

$-\left(4.98 \times 10^{-13}\right) \delta V^{2}+\left(1.9964 \times 10^{-13}\right) \delta V$

$-\left(7.101 \times 10^{-15}\right)$

$c_{4}=-\left(3.1033 \times 10^{-12}\right) \delta V^{9}+\left(4.8112 \times 10^{-11}\right) \delta V^{8}$

$-\left(3.0786 \times 10^{-10}\right) \delta V^{7}+\left(1.0691 \times 10^{-9}\right) \delta V^{6}$

$-\left(2.2718 \times 10^{-9}\right) \delta V^{5}+\left(3.2536 \times 10^{-9}\right) \delta V^{4}$

$-\left(3.3468 \times 10^{-9}\right) \delta V^{3}+\left(2.2308 \times 10^{-9}\right) \delta V^{2}$

$-\left(5.6288 \times 10^{-10}\right) \delta V+\left(2.8565 \times 10^{-11}\right)$

$d_{4}=\left(3.8859 \times 10^{-9}\right) \delta V^{8}-\left(5.6072 \times 10^{-8}\right) \delta V^{7}$

$+\left(3.1746 \times 10^{-7}\right) \delta V^{6}-\left(8.761 \times 10^{-7}\right) \delta V^{5}$

$+\left(1.1436 \times 10^{-6}\right) \delta V^{4}-\left(4.2105 \times 10^{-7}\right) \delta V^{3}$

$-\left(3.5995 \times 10^{-7}\right) \delta V^{2}+\left(1.5266 \times 10^{-7}\right) \delta V$

$-\left(5.3446 \times 10^{-9}\right)$

$e_{4}=-\left(8.8741 \times 10^{-7}\right) \delta V^{9}+\left(1.3434 \times 10^{-5}\right) \delta V^{8}$

$-\left(8.3238 \times 10^{-5}\right) \delta V^{7}+0.00027751 \delta V^{6}$

$-0.0005667 \delta V^{5}+0.00080599 \delta V^{4}-0.00087623 \delta V^{3}$

$+0.00063143 \delta V^{2}-0.00016252 \delta V$

$+\left(8.1928 \times 10^{-6}\right)$

$f_{4}=0.00025791 \delta V^{9}-0.0038432 \delta V^{8}$

$+0.023289 \delta V^{7}-0.075357 \delta V^{6}$

$+0.14916 \delta V^{5}-0.21108 \delta V^{4}+0.23989 \delta V^{3}$

$-0.18269 \delta V^{2}+0.047668 \delta V-0.0023935$ 


$$
\begin{aligned}
& g_{4}=-0.051179 \delta V^{9}+0.74788 \delta V^{8}-4.4036 \delta V^{7} \\
& +13.676 \delta V^{6}-25.859 \delta V^{5}+36.334 \delta V^{4} \\
& -44.11 \delta V^{3}+36.119 \delta V^{2}-9.5874 \delta V \\
& +0.47923 \\
& h_{4}=6.8442 \delta V^{9}-97.59 \delta V^{8}+553.12 \delta V^{7} \\
& -1619.3 \delta V^{6}+2845 \delta V^{5}-3950.4 \delta V^{4} \\
& +5330.8 \delta V^{3}-4813.3 \delta V^{2}+1305.2 \delta V-64.903 \\
& i_{4}=-588.65 \delta V^{9}+8132.2 \delta V^{8}-43715 \delta V^{7} \\
& +\left(1.1669 \times 10^{5}\right) \delta V^{6}-\left(1.7871 \times 10^{5}\right) \delta V^{5} \\
& +\left(2.4225 \times 10^{5}\right) \delta V^{4}-\left(3.9806 \times 10^{5}\right) \delta V^{3} \\
& +\left(4.1292 \times 10^{5}\right) \delta V^{2}-\left(1.15 \times 10^{5}\right) \delta V+5684.5 \\
& j_{4}=29286 \delta V^{9}-\left(3.8778 \times 10^{5}\right) \delta V^{8} \\
& +\left(1.9269 \times 10^{6}\right) \delta V^{7}-\left(4.355 \times 10^{6}\right) \delta V^{6} \\
& +\left(4.6593 \times 10^{6}\right) \delta V^{5}-\left(5.8182 \times 10^{6}\right) \delta V^{4} \\
& +\left(1.5982 \times 10^{7}\right) \delta V^{3}-\left(2.053 \times 10^{7}\right) \delta V^{2} \\
& +\left(5.9161 \times 10^{6}\right) \delta V-\left(2.9038 \times 10^{5}\right) \\
& k_{4}=-\left(6.389 \times 10^{5}\right) \delta V^{9}+\left(7.9757 \times 10^{6}\right) \delta V^{8} \\
& -\left(3.4901 \times 10^{7}\right) \delta V^{7}+\left(5.3272 \times 10^{7}\right) \delta V^{6} \\
& +\left(2.0075 \times 10^{7}\right) \delta V^{5}-\left(5.2271 \times 10^{7}\right) \delta V^{4} \\
& -\left(2.3901 \times 10^{8}\right) \delta V^{3}+\left(4.4785 \times 10^{8}\right) \delta V^{2} \\
& -\left(1.3483 \times 10^{8}\right) \delta V+\left(6.5623 \times 10^{6}\right)
\end{aligned}
$$

\section{References}

1. Prado, A.F.B.A.: Powered Swing-By. J. Guid. Control Dyn. 19(5), 1142-1147 (1996)

2. Da Silva Ferreira, A.F., Prado, A.F.B.A., Winter, O.C.: A numerical study of powered Swing-Bys around the Moon. Adv. Space Res. 56(2), 252-272 (2015)

3. Araujo, R.A.N., Winter, O.C., Prado, A.F.B.A., Vieira Martins, R.: Sphere of influence and gravitational capture radius: a dynamical approach. Mon. Not. R. Astron. Soc. 391(2), 675-684 (2008)

4. Casalino, L., Colasurdo, G., Pastrone, D.: Simple strategy for powered Swing-By. J. Guid. Control Dynam. 22(1), 156159 (1999)

5. Uphoff, C.: The art and science of lunar gravity assist In: AAS/GSFC Symposium, (1989). (AAS 89-170)

6. Nock, K.T., Upholf, C.W.: Satellite aided orbit capture (1979). (AAS/AIAA paper 79-165)

7. Flandro, G.: Fast reconnaissance missions to the outer solar system utilizing energy derived from the gravitational field of jupiter. Astronaut. Acta 12(4), 329-337 (1966)
8. Kohlhase, C.E., Penzo, P.A.: Voyager mission description. Sp. Sci. Rev. 21(2), 77-101 (1977)

9. Byrnes, D.V., D'amario, L.A.: A combined Halley flyby Galileo mission. AIAA/AAS Astrodynamics Conference, San Diego, CA, AIAA paper 82-1462 (1982)

10. Dunham, D., Davis, S.: Optimization of a multiple LunarSwingby trajectory sequence. J. Astronaut. Sci. 33(3), 275288 (1985)

11. Carvell, R.: Ulysses-the Sun from above and below. Space 1, 18-55 (1985)

12. Broucke, R.A., Prado, A.F.B.A.: Planar close encounter trajectories for spacecraft passing near Jupiter. Adv. Sp. Res. 36(3), 561-568 (2005)

13. Prado, A.F.B.A., Broucke, R.A.: Júpiter Swing-By trajectories passing near the Earth. Adv. Astronaut. Sci. 82(Part 2), 1159-1176 (1993). (AAS-AIAA Spaceflight Mechanics Meeting, 3, 22-24)

14. Prado, A.F.B.A., Broucke, R.A.: A classification of SwingBy trajectories using the Moon. Appl. Mech. Rev. 48(11), 138-142 (1995)

15. Sukhanov, A.: Close approach to sun using gravity assists of the inner planets. Acta Astronaut. 45(4-9), 177-185 (1999)

16. Casalino, L., Colasurdo, G., Pasttrone, D.: Optimal lowthrust scape trajectories using gravity assist. J. Guid. Control Dyn. 22(5), 637-642 (1999)

17. Longuski, J.M., Strange, N.J.: Graphical method for gravityassist trajectory design. J Spacecr. Rockets 39(1), 9-16 (2002)

18. McConaghy, T.T., Debban, T.J., Petropulos, A.E., Longuski, J.M.: Design and optimization of low-thrust gravity trajectories with gravity assist. J Spacecr. Rockets 40(3), 380-387 (2003)

19. Heaton, A.F., Strange, N.J., Longuski, J.M.: Automated design of the Europa orbiter tour. J Spacecr. Rockets 39(1), 17-22 (2002)

20. Longman, R.W., Schneider, A.M.: Use of Jupiter's moons for gravity assist. J Spacecr. Rockets 7(5), 570-576 (1970)

21. Lynam, A.E., Kloster, K.W., Longuski, J.M.: Multiplesatellite-aided capture trajectories at Jupiter using the Laplace resonance. Celest. Mech. Dyn. Astron. 109, 59-84 (2011)

22. Longuski, J.M., Williams, S.N.: The last grand tour opportunity to Pluto. J. Astronaut. Sci. 39, 359-365 (1991)

23. Hollister, W.M., Prussing, J.E.: Optimum transfer to Mars via Venus. Astronaut. Acta 12(2), 169-179 (1966)

24. Striepe, S.A., Braun, R.D.: Effects of a Venus Swing-By periapsis burn during an Earth-Mars trajectory. J. Astronaut. Sci. 39(3), 299-312 (1991)

25. Muhonen, D., Davis, S., Dunham, D.: Alternative gravityassist sequences for the ISEE-3 escape trajectory. J. Astronaut. Sci. 33(3), 255-273 (1985)

26. Broucke, R.A.: The celestial mechanics of gravity assist. AIAA/AAS Astrodynamics Conference, Minneapolis, MN, AIAA paper 88-4220 (1988)

27. Araujo, R.A.N., Winter, O.C., Prado, A.F.B.A.: The SwingBy effect in the Vesta-Magnya case. Single and multiple encounters. WSEAS Trans. Syst. 11(6), 187-197 (2012)

28. Okutsu, M., Yam, C.H., Longuski, J.M.: Low-thrust trajectories to jupiter via gravity assists from Venus, Earth and Mars. AIAA/AAS Astrodynamics Specialist Conference, Keystone, CO, AIAA Paper 2006-6745 (2006) 
29. Santos, D.P.S., Prado, A.F.B.A., Casalino, L., Colasurdo, G.: Optimal trajectories towards near-earth-objects using solar electric propulsion (sep) and gravity assisted maneuver. J. Aerosp. Eng. 1(2), 51-64 (2008)

30. McNutt Jr., R.L., Solomon, S.C., Grard, R., Novara, M., Mukai, T.: An international program for mercury exploration: synergy of messenger and bepicolombo. Adv. Sp. Res. 33(12), 2126-2132 (2004)

31. McNutt Jr., R.L., Solomon, S.C., Gold, R.E., Leary, J.C.: The messenger mission to mercury: development history and early mission status. Adv. Sp. Res. 38(4), 564-571 (2006)

32. Grard, R.: Mercury: the messenger and bepicolombo missions a concerted approach to the exploration of the planet. Adv. Sp. Res. 38(4), 563 (2006)

33. Jehn, R., Companys, V., Corral, C., Yárnoz, D.G., Sánchez, N.: Navigating BepiColombo during the weak-stability capture at Mercury. Adv. Sp. Res. 42(8), 1364-1369 (2008)

34. Gomes, V.M., Prado, A.F.B.A.: Swing-by maneuvers for a cloud of particles with planets of the solar system. WSEAS Trans. Appl. Theor. Mech. 3(11), 869-878 (2008)

35. Gomes, V.M., Formiga, J., de Moraes, R.V.: Studying close approaches for a cloud of particles considering atmospheric drag. Math. Probl. Eng. (2013). doi:10.1155/2013/468624
36. Gomes, V.M., Prado, A.F.B.A.: A study of the impact of the initial energy in a close approach of a cloud of particles. WSEAS Trans. Math. 9, 811-820 (2010)

37. Murray, C.D., Dermott, S.F.: Solar System Dynamics, 1st edn. Cambridge University Press, Cambridge (1999)

38. Szebehely, V.: Theory of Orbits. Academic Press, New York (1967)

39. Pourtakdoust, S.H., Sayanjali, M.: Fourth body gravitation effect on the resonance orbit characteristics of the restricted three-body problem. Nonlinear Dyn. 76(2), 955-972 (2014)

40. Zotos, E.E.: Classifying orbits in the restricted three-body problem. Nonlinear Dyn. 82(3), 1233-1250 (2015)

41. Qian, Y.J., Zhang, W., Yang, X.D., Yao, M.H.: Energy analysis and trajectory design for low-energy escaping orbit in Earth-Moon system. Nonlinear Dyn 85(1), 463-478 (2016)

42. Vieira Neto, E., Winter, O.C.: Time analysis for temporary gravitational capture: satellites of Uranus. Astron. J. 122(1), 440-448 (2001) 